\title{
La medición del bienestar económico a través de las macromagnitudes de la contabilidad nacional
}

\section{Ana Belén Miquel Burgos}

"...La justicia prevalecerá en sus días y los justos y los elegidos serán innumerables ante él por los siglos de los siglos"

Libro de 'Enoc (H'e'enukk)

\section{RESUMEN}

La consideración del PIB como un buen indicador del bienestar económico ha quedado superada desde que, en los años setenta, comenzase a cuestionarse su valor omnipotente para reducir la pobreza y lograr el desarroIlo. Como consecuencia, a lo largo de estas décadas han proliferado los trabajos académicos que analizan las limitaciones de dicho agregado para reflejar la realidad. Asimismo, se han creado nuevos indicadores que, a través de distintas aproximaciones, tanto objetivas como subjetivas tratan de perfilar el bienestar económico de las sociedades. Sin embargo, y a pesar de la profusión y calidad académico-científica de muchos de estos trabajos y del aparente consenso de necesidad de mejora, ninguno de ellos ha conseguido allanar el camino para ser utilizado por organismos internacionales, gerentes económicos y políticos y, en definitiva, por la sociedad, como indicador de análisis comparado sincrónico y diacrónico, del mismo modo que lo ha hecho el PIB.

PALABRAS CLAVE: Bienestar económico, desarrollo, pobreza, equidad, Renta Disponible Ajustada. CLAVES ECONLIT: 0110, I310, I320.

Cómo citar este artículo: MIQUEL, B. (2015): "La medición del bienestar económico a través de las macromagnitudes de la contabilidad nacional", CIRIEC-España, Revista de Economía Pública, Social y Cooperativa, 85, 245-286.

Correspondencia: Ana Belén Miquel Burgos, UNED e Instituto de Estudios Fiscales. E-mail: belen.miquel@ief.minhap.es. 


\title{
La mesure du bien-être économique grâce aux données macroéconomiques de la comptabilité nationale
}

RÉSUMÉ : L'utilisation du PIB en tant qu'indicateur fiable du bien-être économique est devenue obsolète à partir des années soixante-dix, lorsqu'on a commencé à remettre en question sa valeur toute puissante permettant de réduire la pauvreté et de promouvoir le développement. En conséquence, les travaux académiques analysant les limites de cet agrégat pour refléter la réalité ont proliféré au cours des décennies suivantes. De nouveaux indicateurs ont ainsi été créés, lesquels tentent, à travers différentes approches tant objectives que subjectives, d'esquisser une image du bien-être économique des sociétés. Cependant, malgré la profusion et la qualité académico-scientifique de nombreux travaux et l'apparent consensus sur la nécessité d'une amélioration, aucun d'eux n'est parvenu à ouvrir la voie pour permettre l'utilisation, de la même manière que le PIB auparavant, de ces indicateurs par les organisations internationales, les gestionnaires économiques et politiques et, en définitive, par la société, en tant qu'indicateurs d'analyse comparative synchronique et diachronique.

MOTS CLÉ : Bien-être économique, développement, pauvreté, équité, revenu disponible ajusté (RDA).

\section{Measuring economic welfare through national accounts macro figures}

\begin{abstract}
The idea that GDP is a good indicator of economic well-being has been superseded since academics in the 1970s started to question its omnipotence for reducing poverty and achieving development. Subsequently, academic studies analysing this aggregate's limitations for reflecting reality have proliferated. Moreover, new indicators have been created through different approaches, both objective and subjective, that have tried to outline the economic welfare of societies. However, despite the profusion and the academic and scientific quality of many of these studies and the apparent consensus on the need for improvement, none of them has managed to smooth the path to adoption - by international organisations, economic and political managers and, ultimately, by society itself - as an indicator of synchronic and diachronic comparative analysis in the way that GDP did.
\end{abstract}

KEY WORDS: Economic welfare, development, poverty, equity, adjusted disposable income. 


\section{1.- Contabilidad nacional y agregados. Evolución conceptual y análisis}

A pesar de los meritorios intentos de medición de la renta realizados durante los siglos anteriores por autores como Sir William Petty $(1665)^{1}$ o Gregory King $(1696)^{2}$, no fue hasta los años 30 y, principalmente, como consecuencia de la crisis surgida en 1929, cuando la necesidad de medir y contabilizar los resultados económicos de las naciones de forma comparable, así como de conocer los efectos de la actuación económica de los gobiernos, se hizo especialmente manifiesta. Lograr estos objetivos obligó a replantearse los fundamentos teóricos de la economía clásica, por lo que algunos autores, como Pigou (1912 y 1920) o Clark (1937), comenzaron a formular estudios sobre la renta nacional.

Es entonces, cuando surgen con gran fuerza las ideas que conformarían la génesis de la economía moderna. John Maynard Keynes con la publicación en 1936 de "Teoría General sobre la ocupación, el interés y el dinero", aportaba una nueva orientación en la que la política económica cobraba un significado distinto, impactando sobre el campo de análisis del ingreso nacional. Con base en dicha teoría y el apoyo del propio Keynes, sus seguidores Stone y Meade, elaboraron en 1941 un informe en el que, como señalan Leon y Marconi (1999) "por primera vez se mostraban los presupuestos como base para la elaboración de un balance económico nacional" y en el que se introducían la renta nacional, los ingresos y gastos de las familias y el gobierno como cuentas nacionales.

Asimismo, Wassily Leontief, a través de su obra "The Structure of the American Economy, 19191939: An Empirical Application of Equilibrium Analysis", (1951) introdujo el modelo económico inputoutput, que permitía analizar y cuantificar las relaciones de los flujos de bienes y servicios intersectoriales, a través del uso de las famosas tablas IO, así como definir la dependencia existente entre ellos, mediante un sistema de ecuaciones lineales, que, posteriormente, se incorporó en la contabilidad nacional de todos los países.

Sin embargo, fue Simon Smith Kuznets, en enero de 1934, quien, en un informe comisionado por el Congreso de Estados Unidos, presentaba la primera medida del ingreso de una economía motivo por el que se le conoce como "padre del PIB"- y que constituyó la base para la creación del primer sistema unificado de contabilidad nacional en los Estados Unidos.

1.- Petty elaboró un sistema de ingreso y consumo en Inglaterra y Gales en su obra Verbum Sapienti.

2.- King desarrolló un esquema que incluía el ingreso nacional, el gasto, los impuestos y la riqueza dentro del Natural and Physical Observations and Conclusions on the State and Condition of England [1696], que no sería publicado hasta 1804 por George Chalmers. 
Finalmente, Stone en 1953 elaboró el primer Sistema de Cuentas Nacionales de aplicación internacional, bajo los auspicios de las Naciones Unidas, al igual que los sucesivos manuales de 1968, 1993 y 2008. Por otra parte, en 1970 se publicaba el primer manual elaborado en el seno de la Comunidad Económica Europea, el denominado "Sistema Europeo de Cuentas Económicas Integradas" (SEC), al que seguirían las versiones de 1979, 1995 y 2010³, de obligada implantación para los países miembros de la UE, según los reglamentos europeos, desde la versión SEC 95.

Las cuentas nacionales pueden definirse como "la presentación, en un marco contable coherente, de toda la información cuantitativa relativa a la actividad económica de la nación" Lequiller y Blades (2009) citan a (Malinvaud, 1973)4. El objetivo principal de las mismas es el de representar, mediante su registro, el funcionamiento económico de una sociedad, articulado en un flujo a través del cual los recursos se trasladan de unos agentes a otros durante un determinado periodo de tiempo, describiendo ese flujo circular y organizando la actividad económica para poder medirla de manera coherente, detallada y bastante completa. Grosso modo, este circuito o flujo circular de la renta, se puede entender como un modelo simplificado que nos muestra cual es esa actuación, es decir, cuál es la actividad económica. Para Lypsey (1996, p. 64), "El flujo circular de la renta es la corriente de pagos entre las empresas nacionales y las economías domésticas del interior o viceversa".

A pesar de que dichos registros y contabilizaciones exigen en muchos de los casos llevar a cabo "retoques" que nos permitan recoger toda la información estadística y ordenarla para poder ser cuantificada, lo cierto es que las cuentas nacionales tienen una gran calidad estadística que, obviamente, variará en función de la disponibilidad de datos, pero que, generalmente, como exponen Lequiller y Blades (2009, p. 342), "son una de las raras excepciones en las que los estadísticos producen tablas (casi) completamente consistentes".

Esta coherencia se consigue a través de la representación de las funciones económicas en una serie de tablas y cuentas que se rigen por el principio de partida doble y que permiten registrar, de manera sintetizada, todas las transacciones llevadas a cabo entre los agentes económicos. Dichas cuentas deber ser completas, coherentes e integradas (SCN 2008) y cada una muestra los recursos que tienen las unidades institucionales, así como el uso que hacen de los mismos, arrastrando los resultados de una a otra, a través de una secuencia de cuentas. De las Cuentas Nacionales se desprenden varias macromagnitudes, o agregados que, como su nombre indica, agregan los saldos de las operaciones que intervienen en el circuito económico, necesarios para poder calcular el valor de la actividad de la economía. No obstante, desde hace décadas, el Producto Interior Bruto se ha convertido en el indicador más relevante de la información económica de los países, ya que su actuación como agregado de toda la actividad productiva, su validez de síntesis y las posibilidades de comparación del dinamismo económico que el mismo permite, le ha llevado a una profusa utilización por Organizaciones Internacionales, instituciones diversas y los propios gestores políticos. Este extenso

3.- Vigente desde el 1 de Septiembre de 2014 en los países de la UE.

4.- Malinvaud Edmond, INSEE (France), Initiation à la comptabilité nationale, Paris : Insee, 1973. 


\section{LA MEDICIÓN DEL BIENESTAR ECONÓMICO A TRAVÉS DE LAS MACROMAGNITUDES DE LA CONTABILIDAD NACIONAL}

uso ha derivado en una ampliación de su finalidad primera, que no es otra que la de proporcionar una medida de la actividad económica de un país, por lo que su paralelismo con la situación de bienestar económico ha sido duramente criticada desde distintos enfoques e idearios que resaltan sus debilidades metodológicas y su falta de adecuación con la medición del bienestar.

En este sentido, suele destacarse el hecho de que el indicador agregue actividades productivas sin tener en cuenta el impacto positivo o negativo para la sociedad o las externalidades negativas diferidas que implicarán menores recursos para las generaciones futuras, como la explotación excesiva de los recursos naturales y la degradación medioambiental. Otra crítica deriva del hecho de que tampoco tenga en cuenta la sostenibilidad financiera ni la depreciación experimentada por el capital, extremo con una solución aparentemente sencilla, a través del uso del PIN en su lugar pero que la heterogeneidad entre los métodos de cálculo del Consumo de Capital Fijo en los distintos países, lleva a desatender. Asimismo, en su cálculo no se contabilizan determinadas actividades que no generan transacciones en el mercado como el autoconsumo, las actividades realizadas en el hogar, la economía de los cuidados y el voluntariado social y su metodología de medición alimenta la creación de burbujas que hacen crecer su valor rápidamente sin tener en cuenta la calidad de ese crecimiento. Probablemente, una de las desventajas más pronunciadas es que el PIB per cápita no tiene en cuenta el reparto del resultado de la producción, de modo que no es posible discernir si el crecimiento del mismo es disfrutado de igual manera o de manera equitativa (en su vertiente vertical) por los ciudadanos de un país.

A pesar de todo, durante las décadas de los cuarenta y los cincuenta se extendió la creencia de que el crecimiento económico o los incrementos del PIB, implicaban el logro de un mayor desarrollo económico, así como la reducción de la desigualdad y la pobreza, equiparando el crecimiento económico con el bienestar de los ciudadanos de un país. Esta teoría se reforzaba, principalmente, a través de las obras de autores de la denominada Economía del Desarrollo como: Rostow (1952), Rosenstein-Rodan (1943) y Nurkse (1953).

En los años setenta comienza a cuestionarse este precepto, y surgen trabajos críticos con el substrato de este ideario como, por ejemplo, la obra pionera de Seers en 1969, "The Meaning of Development", en el que demandaba una noción del desarrollo enfocada hacia "las condiciones universalmente aceptadas para la realización del potencial de la personalidad humana", preponderando la reducción de la pobreza y la desigualdad y el incremento del empleo por encima del crecimiento per se. También el informe encargado al MIT5 por el Club de Roma en 1972, que se tituló "Los límites del crecimiento" y en el que se concluía que "los límites absolutos de crecimiento en la Tierra se alcanzarían en unos cien años de mantenerse el incremento de la población mundial, la industrialización, la contaminación, la producción de alimentos y la explotación de los recursos naturales, al mismo ritmo y sin ninguna variación". Desde entonces, son múltiples los estudios que han apuntado el escaso valor 
del PIB como medida de bienestar económico, especialmente, por su incapacidad para reducir las desigualdades en la sociedad y eliminar la pobreza, surgiendo una fecunda aportación de indicadores alternativos o complementarios al mismo, cuya pretensión es fundamentalmente la de dibujar una imagen fiel o más aproximada de los distintos aspectos que explican la calidad de vida de las sociedades. Entre ellos destaca, especialmente, el Índice de Desarrollo Humano (IDH) publicado por Naciones Unidas (1990), indicador sintético en el que además de las magnitudes monetarias de ingreso, se incorporan índices de educación y esperanza de vida al nacer como valores finalistas de medición del desarrollo de los diferentes países. Otras magnitudes que no han desdeñado la importancia de la dimensión económica en los indicadores sociales son: la Ecuación de Bienestar propuesta por Saint Marc (1971), el Bienestar Económico Neto (MEW) de Tobin y Nordhaus (1973), o el Índice de Bienestar Económico (IEWB) de Osberg y Sharpe (2002). Por otro lado, en los últimos años han aparecido índices desarrollados o respaldados por Organizaciones Internacionales o Fundaciones creadas ad hoc como: el Ahorro Neto Ajustado (ANS) del Banco Mundial (2006), el Índice de Capacidades Básicas (ICB) desarrollado por la Social Watch desde 2007 y con origen en el Indice de Calidad de Vida, el Índice de Progreso Social (IPS) de la Red de Progreso Social (2009). Muchos de ellos, al igual que el ANA están enfocados hacia la sostenibilidad del bienestar, considerando las consecuencias medioambientales del crecimiento económico. Asimismo, en 2012 las Naciones Unidas presentaron en la cumbre Río+20 el Índice de Riqueza Inclusiva (IWI), índice en el que se habían puesto muchas expectativas y del que ya han visto la luz dos informes, pero que no ha gozado del mismo interés que el IDH. Pero, además de indicadores más o menos objetivos, cimentados sobre indicadores de renta, se han venido añadiendo a la lista indicadores basados únicamente en percepciones subjetivas, propios de otras ciencias sociales como la psicología, la sociología e incluso la antropología, tales como el denominado índice de Felicidad Nacional Bruta (FNB) o Felicidad Bruta Interna (FBI) basado en la metodología de Alkire Foster (2006)haciendo trascender al pequeño reino de Bután desde 1972 y que no se basa en medidas cuantitativas sino cualitativas y subjetivas, a través de las cuales pretende obtener una estimación de la felicidad de sus habitantes.

En la misma línea, las Organizaciones Internacionales han desarrollado sus propios indicadores de bienestar. Además del mencionado IDH y el IWI de Naciones Unidas, destacan: el Índice para una Vida Mejor (BLI) de la OCDE (2011), iniciativa que incorpora doce dimensiones que identifica como esenciales para el bienestar humano y el Ahorro Neto Ajustado (ANA) publicado por el Banco Mundial desde 2006, que se obtiene sumando al Ahorro Nacional Neto el gasto en educación y restando el agotamiento de recursos naturales y energéticos. Asimismo, se han venido publicando informes que tratan de dilucidar cómo encontrar mejores indicadores de bienestar o cómo mejorar la medición de los existentes en la contabilidad nacional. En este sentido la Comisión Europea publicó en 2009 un informe dirigido al Consejo y al Parlamento Europeo denominado "Más allá del PIB: Evaluación del progreso en un mundo cambiante", en el que se analizaba la importancia de medir la sostenibilidad medioambiental y la cohesión social, como aspectos de gran importancia en el nuevo contexto político y técnico. Asimismo, Sarkozy encargaba en 2008 a los profesores Stiglitz, Sen y Fitoussi un informe que llevaría por nombre "Informe de la Comisión sobre la Medición del Desarrollo Económico y del Progreso Social", con la misión de reconocer los límites del PIB como indicador de los resultados eco- 


\section{LA MEDICIÓN DEL BIENESTAR ECONÓMICO A TRAVÉS DE LAS MACROMAGNITUDES DE LA CONTABILIDAD NACIONAL}

nómicos y el progreso social, identificando las variables que su medición debería tener en cuenta para evaluar un desarrollo sostenible y que obtuvo como resultado un total de doce recomendaciones de mejora y la invitación a iniciar un debate académico y político, sobre la idoneidad de las herramientas estadísticas utilizadas en la actualidad. Por último, es destacable un tercer estudio elaborado por la OCDE en 2009 denominado "Proyecto global para la medición del progreso de las sociedades", cuya coincidencia cronológica con los anteriores, parece corresponder con los múltiples interrogantes que la primera gran crisis del siglo XXI ha suscitado entre la ciudadanía, los organismos internacionales y los analistas económicos en general, así como en la confirmación de que es necesario un cambio que acomode las herramientas de política económica y sus instrumentos de medición a los cambios y a las demandas de la sociedad.

Sin embargo, y a pesar de la profusión y calidad académico-científica de muchos de estos trabajos y del aparente consenso de necesidad de mejora, ninguno de ellos ha conseguido el uso generalizado por organismos internacionales, gerentes económicos y políticos y, en definitiva, por la sociedad, como indicador de análisis comparado sincrónico y diacrónico, que ha logrado el PIB.

\section{2.- Bienestar económico. Delimitación del término y medición}

Como preámbulo razonable ante cualquier análisis que queramos realizar, es indispensable definir y precisar previamente aquello que pretendemos estudiar. No obstante, debemos partir de la base de que la delimitación conceptual en este caso encierra una complejidad significativa. Decía John Stuart Mill que "los seres humanos tienen facultades más elevadas que los apetitos animales y, una vez se han hecho conscientes de ellas, no consideran como felicidad nada que no incluya su satisfacción".

Desde su aparición sobre la tierra, el hombre se ve avocado a la búsqueda de bienes con los que satisfacer sus necesidades; de hecho, este proceso es inherente a la condición humana, así como a la de otras especies. El hambre, la sed, el frio, la carestía de cobijo y seguridad son algunas de las privaciones básicas que todo ser humano requiere solventar primariamente en el orden maslowiano. Desde el momento en que surge la propiedad, la cuantía de bienes poseídos parece identificarse con una mayor satisfacción, por cuanto permite el acceso a una cantidad de acervos y posibilidades superior para cubrir dichas necesidades. El diccionario de la Real Academia Española (RAE) proporciona como primera definición de bienestar "el conjunto de aquellas cosas que se necesitan para vivir bien" 6 . Este aserto presume que la consecución del bienestar es, por tanto, análoga a la posesión como mecanismo para complacer el instinto de supervivencia. 
No obstante, actualmente, el bienestar se conceptúa más alejado de la visión utilitarista, como un término que engloba una realidad multidimensional extensa, incluyendo variables que, lógicamente, escapan del mero ingreso económico; así las cosas, la libertad, las posibilidades de desarrollo social e individual, la salud, la felicidad, la educación y el entorno, son algunos de los aspectos -aunque se podrían mencionar muchos más- que impactan directamente sobre el nivel de bienestar, en sentido amplio. De hecho, como vemos, algunos de ellos tienen un carácter claramente subjetivo, relacionado tanto con la satisfacción de necesidades personales individuales -ligadas al concepto de utilidad-, como con factores sociales y políticos, basados tanto en aspectos tangibles como percibidos. Por tanto, no es de extrañar que el análisis del bienestar se haya estudiado profusamente desde el terreno de la sociología, la psicología, la filosofía o la antropología.

A pesar de que nos encontremos dentro de la esfera de una ciencia social, si lo que pretendemos es estudiar el bienestar netamente económico y dicho análisis pretende llevarse a cabo a través de indicadores objetivos y cuantificables, circunscribiéndonos a la esfera de esta ciencia podemos abordar una definición de "bienestar" ajustada a la misma, gracias a la delimitación provista por su apellido, concibiendo el "bienestar económico" como "el contexto en el cual los individuos u hogares no sufren privaciones de sus necesidades básicas, existe una coherente igualdad de oportunidades y las inequidades son mínimas, considerando el apéndice de que, además, dicha situación sea sostenible". Debemos tener en cuenta que, la consideración de una situación mínima y coherente en cuanto al grado de igualdad se realiza bajo la premisa de que la situación de equidad total es utópica en economías de mercado, máxime cuando algunos indicadores de bienestar, como por ejemplo la pobreza relativa, se basan en medidas promedio que presuponen la existencia de dicha inequidad.

Una pregunta que ha sido objeto de múltiples respuestas es si las dimensiones abarcadas por el concepto de bienestar económico y, especialmente, la pobreza, deben cuantificarse a través de indicadores construidos a partir del ingreso y la riqueza 0 , si por el contrario, deben disgregarse del valor económico de los bienes y asociarse a otros aspectos, así como a la apreciación subjetiva de los individuos. Independientemente de la indudable utilidad de reconocer su carácter multidimensional, no parece sensato perder de vista la obligación de hacer corresponder, en cierta medida, la delimitación del bienestar económico con la obtención pecuniaria y la tenencia de patrimonio, porque dicha opción implicaría ocultar una realidad incontestable que, no es otra, que el padecimiento de los hombres y mujeres a los que la falta de recursos les impide satisfacer necesidades inherentes a su propia vida. Además, la ampliación de dimensiones abarcadas por el bienestar económico conjuga una cierta relación de dichos factores con la actividad económica de un país. Por ejemplo, Sen explicaba que: "El crecimiento del PIB es menor en los países no democráticos que en los democráticos"7, extremo que también Acemoglu y Robinson (2012) han fundamentado en su ya célebre obra "Por qué fracasan los países: Los orígenes del poder, la prosperidad y la pobreza". De hecho, ya Pigou (1920, p. 10) definía el bienestar económico como "la parte del bienestar social relacionado directa o indirec-

6.- Diccionario de la lengua española, $23^{a}$ edición (2014), Real Academia Española.

7.- Sen, A., "Las distintas caras de la pobreza". Periódico el País. Versión impresa. 30 de agosto de 2000. 


\section{LA MEDICIÓN DEL BIENESTAR ECONÓMICO A TRAVÉS DE LAS MACROMAGNITUDES DE LA CONTABILIDAD NACIONAL}

tamente con la vara de medición del dinero". 80 años después, Osberg y Sharpe $(2003$, p. 8) consideraban que el "bienestar económico de una sociedad depende del consumo total y la acumulación, y en la desigualdad individual e inseguridad que rodean la distribución de los agregados macroeconómicos". No obstante, no debemos olvidar que el ingreso o la riqueza no son una finalidad en sí mismos, sino una mera vía, un medio que nos permite alcanzar la satisfacción de las necesidades ingénitas al ser humano. Aristóteles ya señalaba que "Evidentemente, la riqueza no es el bien que estamos buscando, ya que solamente es útil para otros propósitos y por otros motivos". 8 Además, la exposición anterior no implica la negación de otros factores que se reconocen insertos en el bienestar, en sentido amplio. Sin embargo, creemos que reconocer que la falta de recursos impide lograr una situación de bienestar económico es absolutamente necesario para evitar desviaciones del fundamento central.

Por tanto, partiendo de las sentencias anteriores, creemos que es posible aproximar la mejora del bienestar económico a través de la reducción efectiva de la tasa de pobreza, tanto absoluta como relativa, y la mejora de la equidad en la distribución de los ingresos, añadiendo factores de sostenibilidad, tanto financieros como, especialmente, de los recursos naturales y el medio ambiente.

\section{3.- Pobreza y equidad. Metodología de cálculo}

A pesar de que la pobreza pueda parecer un concepto tristemente cotidiano, delimitar su significado y sobre todo su medición no son cuestiones triviales. No se trata de un término abstracto o de una mera circunstancia que repercuta en las estadísticas de un país, sino de un problema que afecta a la sociedad y, más concretamente, a los hombres, mujeres y niños que sufren cada día esta realidad. No obstante, proceder a su análisis requiere de una generalización que permita establecer una definición y un método de medida que, sin perder la referencia de las circunstancias que rodean esta tragedia social, consiga abstraer las características básicas de la población objeto de estudio y su cuantificación.

Por otro lado, debemos tener en cuenta que no estamos tratando con un término estable, sino con un concepto que ha sufrido multitud de cambios a lo largo de los siglos, conforme a los valores imperantes de cada época y sociedad, siendo redefinido en los últimos tiempos por estudiosos de las ciencias sociales e incluso por Organismos Internacionales.

Existe una gran cantidad de literatura acerca de la pobreza; Adam Smith (1776) ya definía la pobreza como "....) una falta de aquellas necesidades que la costumbre de un país hace que sea indecente, tanto para la gente acomodada como para la de clase más baja, carecer de ellas", resaltando 
que la misma podría manejarse de manera relativa al resto de la sociedad. Si bien, a finales del siglo XIX y principios del XX, los estudios de Booth y Rowntree $(1901)^{9}$, partieron de la situación de subsistencia y desarrollaron el término "umbral de pobreza" como la renta mínima necesaria para sobrevivir; ya a mediados del siglo XX comenzaba a analizarse la pobreza a través del concepto de necesidades básicas, entre las que se incluían no sólo las necesarias para la supervivencia sino también aquellas que proporcionaban un nivel de vida digno.

Destaca fundamentalmente la interrelación entre la pobreza y las capacidades, así como las posibilidades de desarrollo de las mismas, análisis realizado por Sen, "referente inevitable para el análisis económico actual del fenómeno de la pobreza" (Ferullo, 2006, p.10) y que señala que la pobreza no puede determinarse únicamente como una falta de rentas, sino como la necesidad de desplegar las capacidades humanas para conseguir libertad real, considerando la libertad positiva como aquella que permite a una persona desarrollar su capacidad de ser o de hacer ${ }^{10}$, concepción que poco después se postularía como base conceptual del desarrollo humano y que supone la concepción en los años 80 de la pobreza como realidad multifacética inserta en la falta de bienestar, como un problema que incluye multitud de factores económicos y sociales, que se encuentran íntimamente interrelacionados entre sí.

Además, es posible encontrar distintas clasificaciones que atienden a una misma realidad. En función del tipo de información, se define la pobreza objetiva a partir de circunstancias objetivamente mensurables de los hogares ${ }^{11}$, en base a un indicador establecido a priori por el investigador y pobreza subjetiva como aquella que se define a través del nivel de satisfacción percibido por la población. Si consideramos el baremo o referencia que se utiliza para establecer los umbrales de pobreza, diferenciamos entre pobreza relativa, situación de pobreza que se define en función del entorno en que viva el individuo u hogar, y pobreza absoluta, delimitada por la situación de carencia de bienes básicos y/o necesarios para la subsistencia, distinguiendo así entre dos realidades muy diferentes. Finalmente, si atendemos a la dimensión temporal en el análisis de la pobreza, consideraremos la pobreza estática, como aquélla que identifica un momento concreto y pobreza dinámica, la que se estudia como un fenómeno cambiante, comprobando las variaciones en los flujos de entrada y salida a lo largo de un periodo suficientemente largo.

Respecto a la cuantificación de la misma, es destacable que, a pesar de la importancia del problema y la abundante literatura existente, su medición y estudio científico es relativamente actual, remontándose a finales del XIX. De hecho, aunque existen antecedentes sobre estimaciones de

9.- Rowntree realizó un estudio sobre la pobreza en el Condado de York, tratando de establecer una medición de la misma, tomando en consideración un de pobreza que se basaba en requerimientos nutricionales.

10.- SEN, Amartya K. "Desarrollo y libertad", Editorial Planeta, 2000.

11.- Cuando llevamos a cabo mediciones tomando en consideración al "hogar", necesitamos determinar la parte de ingreso que corresponde a cada uno de sus miembros, considerando la existencia de economías de escala y de unidades de consumo equivalentes. Existen multitud de escalas y la elección entre ellas es relevante en la medición. 


\section{LA MEDICIÓN DEL BIENESTAR ECONÓMICO A TRAVÉS DE LAS MACROMAGNITUDES DE LA CONTABILIDAD NACIONAL}

pobreza, señalan Feres y Macero $(2001, \text { p. } 7)^{12}$ citando a Atkinson (1987) que Booth fue "el primero en combinar la observación con un intento sistemático de medición de la extensión del problema". Dicho trabajo consistió en la elaboración de un mapa de pobreza de la ciudad de Londres, realizado entre 1889 y $1897^{13}$, y que contó con varios volúmenes. Los análisis que se han venido realizando desde entonces son numerosos y existe una gran variedad de indicadores y metodologías, debiendo escoger la más adecuada dentro de la región y momento analizado, lo que dependerá principalmente del concepto de pobreza manejado y el enfoque escogido. La medición de la pobreza, según Sen (1976), requiere responder a dos problemas: la identificación de la población objeto de estudio y la construcción de un índice a partir de la información disponible. Generalmente, la solución al primero de ellos es la fijación de un umbral o línea de la pobreza, que sirva de frontera para establecer cuál es la población pobre facilitando su posterior análisis, mientras que las líneas o umbrales de pobreza van asociados al concepto de pobreza utilizado. Para Kakwani (1986, p. 242) la línea de pobreza "debería determinarse sobre la base de lo que la sociedad juzga como el mínimo estándar de vida que todos sus miembros deberían disfrutar". Por su parte, Van Praag, Hagenaars y Van Weeren relacionan las líneas de pobreza con el bienestar y la renta, de hecho Hagenaars (1991, p. 11 y 12) considera que "Cada línea de pobreza puede obtenerse a partir de la definición de bienestar" considerando que "... una línea de pobreza absoluta no está destinada a cambiar con el nivel de vida de la sociedad" y que, sin embargo, "... una línea de pobreza relativa está relacionada de alguna manera con el nivel general de vida de la sociedad".

Para fijar el umbral es necesario escoger con qué variable trabajar, manejando habitualmente criterios económicos a través de variables monetarias, generalmente el ingreso, aunque algunos autores consideran el gasto una variable más estable. Atkinson (1991, p. 5 y 8) defiende que "si la medida se limita a un único índice de recursos económicos, una elección natural puede parecer que sea el consumo total o el gasto, además de los bienes y servicios producidos en el hogar", considerando que el ingreso puede subestimar o sobreestimar el nivel de vida, ya que "variaciones temporales en los ingresos pueden ser suavizadas (aunque tales préstamos pueden ser caros, con graves consecuencias para el futuro)". No obstante, no podemos obviar los problemas estadísticos para la obtención de información de esta variable. Por otra parte, aunque escasamente utilizado, puede plantearse manejar la riqueza neta o patrimonio, cuestión que no es baladí, dada la enorme influencia que tiene en todo el proceso al generar desigualdades de partida, aunque, los estudios suelen centran su atención en los indicadores de flujos monetarios, dejando al margen del análisis la riqueza acumulada por la población objeto de estudio.

Estas líneas de pobreza suelen construirse de manera objetiva, aunque también pueden establecerse líneas subjetivas que se apoyan en la percepción que tienen los hogares o personas sobre

12.- Cita extraida de Atkinson, A. (1987). "Poverty". En: Eatwell, Milgate, Newman (eds.), The New Palgrave: A Dictionary of Economics. Macmillan Press, London.

13.- Existen dos ediciones anteriores: Booth, C. "Life and Labour of the People" 1st Edition Vol. I (1889), Vol II (1891). Y una tercera edición con 9 volúmenes: "Life and Labour of the People in London", 3th Edition, publicados entre 1892 y 1897. 
cuáles son sus propias necesidades y cuál es su situación económica y social, para lo cual, se realizan encuestas directas a la propia población, preguntando sobre su situación económica o aspectos del bienestar, para que ellos respondan en función de su propio discernimiento. Este método se considera un buen mecanismo para mejorar las políticas públicas, a partir del mayor conocimiento de las percepciones y expectativas de la población, así como las prioridades que éstos hagan de las mismas. De este modo las metodologías subjetivas de mayor utilización son:

La Línea de Kapteyn obtiene información a través de una encuesta directa en la que se pregunta sobre los ingresos mensuales mínimos necesarios para llegar a fin de mes, dependiendo del tamaño del propio hogar y del nivel de ingresos del mismo. Con base en esta teoría, se construye un modelo a través de una ecuación matemática tipo Cobb-Douglas:

$$
\log Y *(i, t)=\alpha+\beta_{1} \log Y(i, t)+\beta_{2} \log N(i, t)+u[i, t]
$$

Donde: $\boldsymbol{Y} *(i, t)$ es la respuesta o ingreso mínimo que según el hogar es necesario para llegar a fin de mes. $Y(i, t)$ la renta real del hogar i en el momento $t$. $N(i, t)$, el tamaño o número de miembros de dicho hogar. $\boldsymbol{u}[\boldsymbol{i}, \boldsymbol{t}]$ es una perturbación aleatoria con esperanza cero.

Promediando a través de la distribución de los hogares e igualando $Y *(t)=Y(t)$ obtenemos el umbral de pobreza o mínimo general de necesidad familiar $M(t)$ :

$$
\log M(t)=\frac{1}{1-\beta_{1}}\left[\alpha+\beta_{2} \log N(t)\right]
$$

Determinando un umbral de pobreza común, de modo que, para obtener el umbral individual será necesario dividir dicha ecuación por el tamaño del hogar, de forma que, la curva se desplaza hacia arriba conforme aumenta el tamaño del hogar $N(i, t)$.

Por su parte, la Línea de pobreza de Leyden $(L P L)$ se basa en la Función de Bienestar de la Renta (WFI), que se deriva de la Encuesta de Evaluación de la Renta (IEQ), considerando pobres los hogares con ingresos inferiores al nivel de utilidad mínimo que se mide a través de la Función de Bienestar de la Renta (WFI), cuya determinación se obtiene a través de las opiniones subjetivas resultantes de una encuesta, la IEQ, así como de otras informaciones complementarias. Para ello, se pregunta con qué situación se puede asociar "aproximadamente" el ingreso del hogar, permitiendo escoger entre: muy malo, malo, muy deficiente, deficiente, bueno y muy bueno. Cada una de estas respuestas comprende un intervalo acotado entre 0 y 1 , cuyo valor se estima, con las seis cifras proporcionadas por cada hogar, obteniendo una función de utilidad cardinal de la renta, (WFI) log-lineal. La mayor complejidad que encierra esta técnica se compensa con estimaciones más precisas, al acotar las cifras y respuestas de las familias. 


\section{LA MEDICIÓN DEL BIENESTAR ECONÓMICO A TRAVÉS DE LAS MACROMAGNITUDES DE LA CONTABILIDAD NACIONAL}

Una tercera línea es la proporcionada por la Metodología del Centro de Política Social de Amberes (C.S.P) o línea de Deeleck, basada en la encuesta Minq (Encuesta sobre la renta mínima), que realiza comparaciones entre el grado de bienestar de los hogares, en función de una pregunta especial de actitud sobre las posibilidades de cubrir los gastos del hogar con los ingresos. De todas las respuestas (con gran dificultad, con dificultad, con cierta dificultad, más bien fácilmente, fácilmente o muy fácilmente) se podría escoger solo una. Posteriormente Deeleck seleccionaba únicamente la respuesta "con cierta dificultad" y obviaba las demás, de manera que a partir de esta categoría obtenía el ingreso límite inferior que le permitía establecer el umbral de pobreza a través del ingreso familiar del periodo $\boldsymbol{Y}_{\boldsymbol{c}}$ y el declarado como ingreso mínimo $\boldsymbol{C}_{\boldsymbol{M I N}}$ en la encuesta. Precisamente las críticas que han surgido en torno a esta línea, se basan en la reducción excesiva del tamaño de la muestra, al escoger únicamente esa respuesta.

Las líneas de pobreza relativa, tienen en cuenta la relatividad propia del tiempo y del lugar de estudio, de manera que una persona será relativamente pobre respecto a la sociedad en la que vive si se encuentra por debajo del umbral o mínimo establecido, que generalmente se construye a partir de una medida promedio del ingreso, como la media o la mediana de la distribución total o también otros cuantiles (deciles, ventiles, etc.).

Asimismo, la delimitación tradicional de pobreza se realiza a través de líneas de pobreza absoluta, las cuales marcan "el valor de los recursos necesarios para mantener un mínimo bienestar" (INE, 2005), es decir, la cuantía económica de aquellos productos esenciales para lograr la satisfacción de las necesidades básicas.

Por último, existen líneas de pobreza multidimensionales, concepto relativamente moderno, que amplía la selección de indicadores utilizados, de modo que, para establecer una clasificación de la población que identifique la pobreza, se tienen en cuenta otras variables además de las económicas como: la educación, el medio ambiente, el ocio o la sanidad. Esta moderna visión se basa en el enfoque de Sen cuyos estudios pueden considerarse como uno de los factores fundamentales para este cambio de actitud en el establecimiento del término, con aseveraciones como: "utilizar una línea de pobreza que no varíe entre las personas, puede ser muy equivocado para identificar y evaluar la pobreza" Sen (1987, p. 22) y "no existe una correspondencia estrecha entre 1) la pobreza vista como escasez del ingreso, y 2) la pobreza vista como incapacidad para satisfacer algunas necesidades elementales y esenciales" Ponce (2013, p. 10) cita a Sen (2000, p. 239).

Este carácter multidimensional también ha sido incorporado en 2001 por el Banco Mundial, "La visión, ya establecida, de pobreza engloba no solo bajo ingreso y consumo, sino también bajo rendimiento en educación, salud, nutrición y otras áreas del desarrollo humano"14. 
Asimismo, la Organización de Naciones Unidas para el Desarrollo ha adoptado un enfoque multifacético, presentando, en colaboración con la Universidad de Oxford ${ }^{15}$, en 2010 -su 20 aniversariodel "Índice de Pobreza Multidimensional" (MPI), que supera la incapacidad para reflejar todos los aspectos que ayudan a conceptualizar la pobreza de los índices complementarios al IDH anteriores, "Índices de Pobreza Humana" (IPH 1 y 2) ${ }^{16}$, y trata de obtener una visión más amplia y exacta de esta situación. De este modo, una persona se consideraría multidimensional pobre, si los indicadores ponderados en los que él o ella padeciesen carencias sumaran, al menos, un 33\%. Para el cálculo de las privaciones experimentadas en cada hogar se tiene en cuenta la suma ponderada de las distintas dimensiones, asignando una puntuación a cada una de ellas hasta alcanzar un máximo del $100 \%$, con una ponderación del 33,3\% para cada aspecto y sumando las distintas puntuaciones, lo que permite obtener la privación total del hogar " $c$ ", y comparar el valor obtenido con los umbrales establecidos, de tal manera que el hogar se considerará:

Si $\boldsymbol{c} \geq 33,3 \% \Rightarrow$ multidimensionalmente pobres.

Si $20 \% \leq c \leq 33,3 \% \Rightarrow$ vulnerables o en riesgo de caer en la pobreza multidimensional.

Si $\boldsymbol{c} \geq 50 \% \Rightarrow$ dentro de la pobreza multidimensionalmente extrema.

Finalmente, la obtención del valor IPM se obtiene a través de la agregación geométrica de dos medidas: la tasa de la incidencia multidimensional (H) y la intensidad (o amplitud) de la pobreza (A).

$$
H=\frac{q}{n} \text { y } A=\frac{\sum^{q}{ }_{1} c}{q}
$$

Donde: $\boldsymbol{q}$ es el número de personas multidimensionalmente pobres, $\boldsymbol{n}$ es la población total y $\boldsymbol{A}$ refleja la proporción de los indicadores de los componentes ponderados " $d$ " en los que, en promedio, los pobres están sujetos a privaciones.

Pero, a pesar de su interés académico, los indicadores mixtos y multidimensionales plantean un problema de cuantificación al presentar mayor complejidad y dificultades adicionales de obtención de los valores de las dimensiones, así como la falta de consenso sobre que parámetros utilizar, que nivel mínimo determinar o qué cantidad de indicadores, siendo complicada su agregación y comparabilidad, si cada aspecto ponderado avanza en un sentido diferente.

Un aspecto fundamental es la elección del índice con el que llevar a cabo la medición, lo que permite obtener un valor resultante que representa la magnitud de la pobreza (incidencia) y posteriormente evaluar el grado (o intensidad). En este sentido, se deben tener en cuenta una serie de

15.- Oxford Poverty and Human Development Initiative [OPHI]. http://www.ophi.org.uk/

16. - La UPND dividía a los países para su estudio en dos grupos en función del nivel de desarrollo económico: desarrollados (IPH 2) y en desarrollo (IPH1). 


\section{LA MEDICIÓN DEL BIENESTAR ECONÓMICO A TRAVÉS DE LAS MACROMAGNITUDES DE LA CONTABILIDAD NACIONAL}

propiedades cuyo cumplimiento, por los índices de pobreza que se establezcan, se formula como deseable o exigible. Sen (1976) fue el primero en establecer algunos de estos axiomas, desarrollando en primer lugar los axiomas de monotonicidad (débil o fuerte), que en síntesis presume que el índice debe aumentar cuando la renta de los pobres disminuya y posteriormente el de transferencias ${ }^{17}$, de modo que si éstas son progresivas disminuyen la desigualdad, es decir, las transferencias de una persona pobre a otra persona de mayor ingreso debe incrementar el índice. Asimismo se consideran: el axioma de dominio del índice, que le hace depender únicamente de las rentas de los pobres, (Kakwani, 1980), sensibilidad a las transferencias, según el cual las transferencias de una persona pobre a otra con menor ingreso aumenta el valor del índice, simetría, por el que los cambios de posición entre dos individuos no alteran el índice y enfoque, que supone la independencia respecto de los ingresos de los no pobres; Foster y Shorrocks (1991), descomponibilidad, permite expresar la pobreza total a través de la media ponderada de las pobrezas de los distintos subgrupos, consistencia por subgrupos: si un grupo de personas aumenta su pobreza, aumentará el valor de pobreza total. Foster et al. (1984) explican el Axioma de Monotonía de subgrupos, condición necesaria para la descomponibilidad aditiva expresada por los mismos autores. El cual exige que las variaciones en los ingresos de un subgrupo que les empobrezcan sin cambiar su participación en el total de la población, aumenten el valor del índice. Esta exposición no es exhaustiva, existiendo además algunos otros indicadores menos mencionados. Lo cierto es que la utilización de uno u otro índice más que del cumplimiento del enfoque axiomático, por lo general, se realizará finalmente en función de la valoración del investigador añadiendo su propia subjetividad en la elección.

El indicador de mayor simplicidad y más frecuentemente utilizado es el Head-count ratio $(\mathrm{H}) 0$ índice de recuento, el cual se define como la proporción de pobres que hay en la población total y que se obtiene dividiendo el número de hogares que quedan por debajo del umbral de pobreza establecido " $q$ " por el total de hogares estudiados " $n$ ". Siendo: $X=\left(X_{1}, X_{2}, \ldots, X_{n}\right)$ el vector indicativo de la situación económica de $n$ hogares que constituyen la población " $N$ ” y $Z$, el umbral de pobreza considerado. Obtenemos el índice:

$$
H(Z, X)=\frac{q}{n}
$$

A pesar de su facilidad de cálculo, $\mathrm{H}$ presenta una serie de desventajas que deben tenerse en cuenta. Sen (1976, p. 219) recuerda que la información provista por este índice es incompleta, ya que, se trata de un índice insensible a la intensidad de la pobreza y no tiene en cuenta el efecto de las transferencias entre una persona por debajo de la línea de pobreza a otra más rica, quebrantando claramente los axiomas de monotonicidad y de transferencia.

Otro índice bastante extendido es el Income gap ratio (I) o índice de brecha del ingreso, el cual calcula el consumo total necesario para salir de la condición de pobreza y la media de los desniveles 
relativos de la misma ${ }^{18}$. Su expresión matemática utiliza lo que denominamos Gap o Brecha de pobreza Poverty Gap (PG), la cual se determina como la distancia, en términos de renta, que separa a los individuos pobres de la línea de la pobreza. Siendo $\boldsymbol{Z}$ el valor que toma la línea de pobreza y $\boldsymbol{X}_{\boldsymbol{i}}$ el ingreso o consumo (según el indicador establecido) de los hogares o individuos que se encuentran por debajo del umbral. La brecha o gap de pobreza se calcula como:

$$
P G=\sum_{i=1}^{q}\left[Z-X_{i}\right]
$$

Si relativizamos el índice para el total de consumo o ingreso resultante en el supuesto de que todos los individuos pobres consumieran o ingresaran exactamente el valor de la línea de pobreza, es decir $q^{\mathbf{z}}$, obtenemos el Income Gap Ratio que, expresado en función de la media del consumo o ingresos de los hogares que se encuentran por debajo del umbral de pobreza, se define como:

$$
\mu_{p}: I(Z, X)=1-\left(\mu_{p} / z\right)
$$

La utilización de este índice permite incorporar los aspectos dependientes del grado de pobreza, pero es indiferente a la proporción que representa la población pobre en el total y no permite conocer la desigualdad en la distribución de la renta.

Por su parte, el Poverty Gap Ratio (HI) toma en consideración los anteriores para tener en cuenta tanto la población afectada como el grado de pobreza, definiéndose como la cantidad de renta o consumo necesario para poder situar a todos los pobres por encima del umbral de la pobreza. Siendo $n \boldsymbol{Z}$ el ingreso o gasto total, para que todos los individuos lleguen exactamente al valor de la línea de pobreza.

$$
H I=\frac{\sum_{1}^{q}\left[Z-X_{i}\right]}{n Z}
$$

Desarrollándolo, permite llegar a una expresión en función de los índices anteriores, $\mathrm{He} \mathrm{I}$ :

$$
H I=\frac{\sum_{1}^{q}\left[Z-X_{i}\right]}{n Z}=\frac{q}{n}-\left(\frac{\sum_{1}^{q}\left[X_{i}\right]}{n Z}\right)=H\left(1-\frac{\sum_{1}^{q}\left[x_{i}\right]}{q Z}\right)=H \times I
$$




\section{LA MEDICIÓN DEL BIENESTAR ECONÓMICO A TRAVÉS DE LAS MACROMAGNITUDES DE LA CONTABILIDAD NACIONAL}

El Índice de Sen recoge información sobre la incidencia de la pobreza la intensidad y la desigualdad (a través del Coeficiente de Gini). Se calcula como la suma ponderada (en función de la posición relativa de cada uno de ellos entre la población pobre), de las brechas individuales de ingreso 0 consumo de los hogares o individuos pobres y se formula como:

$$
S=H\left[I+(1-I) G_{q}\right] \frac{q}{q-1}
$$

Si el número de hogares pobres es lo suficientemente grande, puede aproximarse a:

$$
S(X, Z)=H\left[I+(1-I) G_{q}\right]
$$

Este índice satisface los tres axiomas propuestos por el propio Sen, pero no el de transferencias regresivas ni descomponibilidad aditiva.

La Familia de Índices Foster, Green y Thorbecke (FGT) cumple un mayor número de axiomas, proporcionando una medida de sensibilidad distinta a cada uno de los componentes o déficits que presentan los individuos para alcanzar la línea de pobreza. La sensibilidad del índice o de aversión a la pobreza es $\alpha$, que tornará valores más elevados cuanto mayor sea la ponderación que se asigne a los individuos u hogares con déficits mayores en el cómputo.

$$
F G T(\alpha)={ }_{n}^{1}\left(\frac{\sum_{i=1}^{q}\left(z-x_{i}\right)}{Z}\right)^{\alpha} \forall \alpha \geq 0
$$

Este índice puede considerarse como una generalización de índices anteriores (Gradín y Del Rio, 2001) de modo que:

Si $\alpha=0$, el índice $\mathrm{FGT}(0)=\mathrm{H}$, con lo que coincide con el Headcount ratio.

Si $\alpha=1$, el índice FGT (1) = HI, es decir, que coincidirá con el Poverty gap ratio.

Si $\alpha=2$, el índice FGT (2) $\Rightarrow S$, se aproxima al índice de Sen.

Por último, cuando $\alpha \Rightarrow \infty$, da únicamente valor a la brecha del individuo más pobre, con lo que esta será la única situación reflejada en el índice.

El Índice de Hagenaars se basa en la evaluación de la pérdida de bienestar social cuando no hay una adecuada distribución de la renta. Matemáticamente puede expresarse como:

$$
H A G(y, z)=H \frac{\ln z-\ln \mu_{p}^{*}}{\ln z}
$$

siendo: $\mu_{p}^{*}$ la media geométrica de la renta de los hogares pobres. 
Finalmente, existe un tipo de medición distinto a los índices, las curvas TIP de pobreza (las tres "l" de la pobreza: incidencia, inequidad e intensidad), denominadas así por Jenkins y Lambert (1997), que se construyen de manera equivalente a la curva de Lorenz, describiendo gráficamente las dimensiones mencionadas, de modo que se representan en el eje horizontal la proporción de pobres y en el vertical las brechas de pobreza acumuladas, mostrando el nivel de desigualdad relativa en la distribución de la población pobre, sin depender estrictamente de las líneas o índices de pobreza.

Uno de los aspectos más criticados de la cuantificación del bienestar a través del simple incremento de las cifras de PIB per cápita, es el hecho de que dicho aumento no garantice el bienestar económico de todos, cuando las bondades de la mayor disposición de renta no pueden ser disfrutadas por la población de manera proporcional. Efectivamente, en primer lugar debemos tener en cuenta que, generalmente, la riqueza de un país no se reparte de manera equitativa entre todas las capas sociales, pero además, aún en el caso de que dichos frutos se distribuyesen por igual entre todos los estratos de renta, tampoco implicaría una disminución de la pobreza en términos relativos o una mejora de la equidad en dicha nación, puesto que una distribución totalmente igualitaria del incremento del PIB no disminuye la pobreza ni la inequidad en una sola décima, pues la distribución original permanece inalterada. Por tanto una distribución equitativa sería aquella que se plasmase considerando las dos vertientes de la equidad: horizontal, tratando por igual a aquellos que se encuentran en la misma situación de partida, y vertical, con una distribución progresivamente mayor hacia aquellos con menores ingresos o riquezas iniciales, lo que garantizaría el progreso respecto del escenario preliminar.

Tradicionalmente, el estudio de la equidad se puede abordar desde cuatro enfoques diferentes, que suponen distinguir entre: su distribución funcional, entre los factores productivos ${ }^{19}$; personal, analizando la renta disponible que llega a los hogares; sectorial, por ramas de actividad; e incluso territorial, aspecto de gran interés para detectar las diferencias en la distribución de la misma entre territorios 0 áreas geográficas, políticas 0 administrativas.

Pero, además del enfoque de distribución, es necesario seleccionar la metodología más adecuada para llevar a cabo un análisis cuantitativo que nos permita conocer el grado de desigualdad de la población y su estratificación en función del reparto de riqueza entre sus miembros. El ingreso es generalmente la variable utilizada, a pesar de que puede presentar ciertos problemas derivados del sesgo de las encuestas. Sin embargo, como veíamos, la riqueza neta o patrimonio puede generar mayor fidelidad, en primer lugar por ser una variable stock, presentando atributos de permanencia y de generación de nuevos recursos, lo que aumenta su concentración y ayuda a explicar la diferencias iniciales y acumulativas. En este sentido, los resultados del estudio realizado por Davies, Sandström, Shorrocks y Wolff (2008) evidencia a través de una muestra de 27 países que, el coeficiente de Gini, calculado con datos de riqueza, se incrementaba en todos ellos notablemente respecto a los resultados del coe- 


\section{LA MEDICIÓN DEL BIENESTAR ECONÓMICO A TRAVÉS DE LAS MACROMAGNITUDES DE LA CONTABILIDAD NACIONAL}

ficiente según la distribución de ingresos. Asimismo, Milanovic (2005) calculaba dicho coeficiente como valor global, midiendo la desigualdad en riqueza e ingresos y de nuevo obtenía que el primer valor excedía del primero, aunque para el conjunto del mundo esta diferencia, que era del 10\%, se reducía respecto al estudio por países de Davies et al.

Respecto a la metodología de medición, además de las medidas estadísticas como la varianza o la desviación típica, la construcción de indicadores de desigualdad se realiza a partir de estadísticos que tratan de medir por ejemplo la dispersión de la variable observada o la distancia respecto a la equidistribución. En este sentido, las medidas más características para medir la distribución del ingreso son:

- La "Curva de Lorenz", representa gráficamente la desigualdad de la distribución de la renta y relaciona el porcentaje acumulado del ingreso total de una sociedad con el porcentaje de la población receptora, de modo que, cuanto más cercana se encuentra la distribución del ingreso a la bisetriz del cuadro formado por ambas variables mayor igualdad en la distribución posee dicha sociedad.

$$
L[p]=\frac{n \int_{0}^{R[p]} r f(r) d r}{n \int_{0}^{\infty} r f(r) d r}=\frac{1}{m} \int_{0}^{R[p]} r f(r) d r
$$

- El "Coeficiente de Gini" se puede calcular como proporción de las áreas resultantes la curva anterior midiendo la superficie entre la curva y la línea de hipotética equidad absoluta, obteniendo un porcentaje entre 0 (equidad absoluta) y 1. $G=\sum_{k=1}^{n-1}\left(X_{k+1}-X_{k}\right)\left(Y_{k+1}+Y_{k}\right)$

Este es el indicador más utilizado, tanto por su sencillo cálculo e interpretación, como por el hecho de que sus propiedades estadísticas son más conocidas, permitiendo comprobar la robustez de los cambios que se generan en el nivel de equidad Medina y Galván (2008, p. 14) citan a Wodon y Yitzhaki, (2002).

- Otro coeficiente que también es utilizado con relativa frecuencia es el Índice de Theil, que se basa en las medidas de la entropía de Shannon, a través de la cual mide el grado de desigualdad en la distribución de la renta, definiéndose como la información esperada del mensaje que transforma porcentajes poblacionales en participaciones de ingresos.

$$
T=\sum_{i=1}^{n} y_{i} \log \left[Y_{i} / P_{i}\right]
$$

- Donde $Y_{i} / P_{i}$ será la renta per-cápita del i-ésimo conjunto deflactada por la renta per-cápita de la población total. Puede alcanzar valores entre 0 y 1 , según sea más o menos equitativa la distribución y su principal ventaja reside en las propiedades de descomposición que permiten analizar la rela- 
ción entre la desigualdad de una variable y una serie de variables explicativas Altimir y Piñera (1979, p. 207).

- Por último, destacamos un índice que, al igual que el Índice de Dalton, se basa en una función de utilidad, pero que supera los defectos del anterior, el Índice de Atkinson (1970): $\boldsymbol{A}=1-\boldsymbol{x}_{\boldsymbol{e}} / \boldsymbol{\mu}$

El cual, se define a través de la renta igualitaria equivalente, $X e$, que es el ingreso que distribuido equitativamente proporciona el mismo nivel de utilidad que la distribución existente. Además, este índice exige desarrollar una función de utilidad de bienestar social de aversión constante a la desigualdad, la cual se establece como:

$$
U(X)=\left\{\begin{array} { c c } 
{ a + \frac { b x _ { i } ^ { 1 - \varepsilon } } { 1 - \varepsilon } ; \quad } & { \text { si } \varepsilon > 0 \neq 1 } \\
{ b L _ { n } ( X _ { i } ) ; } & { \text { si } \varepsilon = 1 }
\end{array} \quad \text { Derivando obtenemos: } A \left\{\begin{array}{cc}
1-\left[\sum \frac{X_{i}{ }^{1-\varepsilon}}{N}\right]^{\frac{1}{1-\varepsilon}} ; & \text { si } \varepsilon>0 \neq 1 \\
\operatorname{Exp}\left[\sum \frac{\ln x_{i}}{N}\right] ; & \text { si } \varepsilon=1
\end{array}\right.\right.
$$

$\mathrm{U}(\mathrm{X})$ es una función cóncava y homotética en la que $\varepsilon>0$ es el parámetro de aversión a la desigualdad, de modo que $\varepsilon=0$ implica indiferencia o neutralidad ante la desigualdad y a medida que aumenta el valor de $\varepsilon$ aumenta la aversión relativa de la misma.

No obstante, nuevamente nos encontramos frente al criterio arbitrario del investigador y la disponibilidad de datos a la hora de escoger el indicador más adecuado 0 a la necesidad de utilizar varias de las opciones comentadas para complementar el análisis.

\section{4.- Impacto del crecimiento económico en la reducción de la pobreza y la inequidad}

¿Es el crecimiento económico capaz de reducir la pobreza extrema por si solo? ¿Y la pobreza relativa? ¿Y la inequidad? ¿Somos capaces de seguir creciendo sin acabar con el planeta? ¿Y de reducir el efecto de los ciclos económicos? En definitiva, ¿crecer implica mejorar el bienestar económico de una sociedad?

Como comprobábamos, a partir de los años setenta, se asiste al destronamiento del todopoderoso crecimiento económico como panacea para acabar con la pobreza y la inequidad, surgiendo una fecunda aportación académico-científica, que actúa como soporte del intenso debate sobre la existencia o no de una relación sistemática entre crecimiento y bienestar económico. Algunas de las obras tratan de discernir si incrementar el producto de un país ayuda a reducir la pobreza en el mismo 0 


\section{LA MEDICIÓN DEL BIENESTAR ECONÓMICO A TRAVÉS DE LAS MACROMAGNITUDES DE LA CONTABILIDAD NACIONAL}

cuanto se reduce la pobreza como consecuencia de dicho aumento, así, Ahluwalia et al. (1979) Jain y Tendulkar (1990), Ravallion y Huppi (1991), Kakwani (1993), Bell y Rich, (1994) Ravallion y Chen (1997), Ravallion y Datt (1999), Barro (1999) Dollar y Kraay, (2000), y Bourguignon, (2002) son algunas contribuciones interesantes sobre dicha relación. Los resultados a los que llegan difieren de unos a otros, principalmente en función del tipo de pobreza, la región o el periodo que tomen en consideración en dicho análisis. Por ejemplo, el estudio de Barro (1999) o el que Dollar y Kray (2001) realizaron para el Banco Mundial estudian cómo afecta un mayor dinamismo económico sobre las rentas más bajas de la población, pobres relativos, en función de la renta per cápita del país, lo que se adentra en el terreno de la inequidad y la distribución. En este sentido, mucho más profusa y antigua es la contribución teórica y empírica sobre la relación causal del crecimiento con la distribución de sus frutos. Dentro de este campo cabe destacar los trabajos de Adelman y Morris, (1973), Jain (1975), Robinson (1976), Fields (1980), Saith (1983), Tsaklogou (1988), Ram (1988), Bourguignon y Morrison (1990), Anand y Kanbur (1993 b) Fields y Jakubson (1994) Bourguignon (1994) Clarke (1995), Ravallion (1995), Deininger (1998), Aghion et al (1999) y Banerjee y Duflo (2000). Pero, sin duda, merece observación aparte la aportación de Kuznets, que muchos de los autores comentados han utilizado como base de su análisis, y que supone una relación positiva del incremento del ingreso con la desigualdad en los primeros estadios del crecimiento hasta alcanzar un punto de retorno -previsiblemente con la consecución de la madurez económica- y a partir de ese punto de inequidad máxima, una relación inversa entre ambos, esbozando una curva en forma de U invertida. En 1955, Simon Kuznets lanzaba por primera vez esta hipótesis como motivo del discurso presidencial de la American Economic Association pero la escasa contrastación empírica, fundamentada en datos transversales de unos pocos países, llevaron al autor a continuar su investigación, hasta que en 1963 publicase "Quantitative Aspects of the Economic Growth of Nations" esta vez con una base estadística mayor y conformando la que posiblemente sea la teoría más relevante en este terreno. Kuznets planteaba que dicha relación podría deberse al trasvase de mano de obra de un sector a otro, motivado por el crecimiento, así como a la implantación de nuevas tecnologías. Desde entonces, la aportación analítica y crítica, asentada en esta teoría, ha sido fecunda y diversa; tanto de aquellos que o bien la han desacreditado, basándose en los supuestos restrictivos de partida y su débil base empírica, o bien han refutado los resultados obtenidos por Kuznets tras su propio análisis empírico, como la de otros autores que sí han corroborado dicha hipótesis, obteniendo corolarios similares.

Los primeros estudios que se realizaron para contrastar esta hipótesis fueron igualmente de corte transversal. Así, apoyaban la hipótesis de la curva en U invertida, Ahluwalia (1976), quién llevó a cabo un análisis para 62 países a través de varios aspectos que enlazaba con el desarrollo económico, Paukert (1973), que comparaba promedios del coeficiente de Gini por grupos de países en función de su PIB, y Ram (1988) y Braulke (1983). Igualmente Robinson (1976), Knight (1976), Dawson (1997) y Easterly (2003) son algunos de los autores que dentro de un abanico mucho más amplio han dado validez a la misma. Sin embargo, otros trabajos han obtenido relaciones funcionales diferentes, en forma de "U" no invertida o de "L", e incluso otros autores han negado cualquier tipo de correspondencia entre crecimiento e inequidad en la distribución. Así las cosas, Anand y Kanbur (1993, a), consideraban que no se podía establecer una relación sistemática concreta, aludiendo a la insuficiencia 
de datos para poder establecer una teoría al respecto. Otros estudios que rechazan esta hipótesis son por ejemplo los de Saith (1983), Fields (1995), Ravallion (1995) Ravallion y Chen (1997), Barro (1999) o Deininger y Squire (1998), que, con distintos tipos de muestras o utilizando datos de panel, rechazaron con sus conclusiones la teoría de Kuznets. Generalmente, gran parte de la aportación más reciente coincide en señalar los problemas que plantean la falta de datos, sobre todo de tipo longitudinal, en países de bajo ingreso, siendo necesario en muchos de los casos imputar datos perdidos 0 realizar estudios transversales con datos cronológicos no homogéneos, lo que deriva en una menor calidad de las conclusiones obtenidas.

Por otra parte, también han comenzado a surgir interesantes aportaciones que describen la relación causal crecimiento-equidad pero en sentido opuesto, enfocando la discusión en los efectos de la distribución del ingreso o la riqueza y la pobreza sobre el incremento del PIB, tratando de dilucidar si la inequidad produce un impacto negativo sobre el crecimiento de los países, presunción que se ha visto respaldada por las nuevas teorías del crecimiento endógeno, que desde los años 80 , reabren el debate del crecimiento y el desarrollo y cuestionan las teorías neoclásicas. Esta nueva corriente se basa en las aportaciones de autores como Romer (1986), Lucas (1988), Aghion y Howitt, (1998) y Barro (1990), considerando que el empuje al progreso económico se provoca de manera endógena y no exógena, especialmente a través de la acumulación de capital humano, el capital físico y la innovación, de modo que la inequidad impediría dicho proceso acumulativo, afectando asimismo a la productividad, otro de los aspectos claves para el desarrollo económico. Por tanto, son varios los estudios que afirman que una mayor equidad contribuiría positivamente a impulsar el crecimiento económico, Ros (2004), Alesina y Rodrik (1994), Alesina y Perotti (1994), Birsdall, Ross y Sabot (1995) o Clarke (1995), son algunos ejemplos de este nuevo enfoque, que llevan a una importante discusión académica en el terreno del crecimiento y la equidad, y la relación causa-efecto entre ambos.

Por último, también podemos encontrar algunos autores que han estudiado la trilogía crecimiento, pobreza y equidad y la relación entre las tres variables, entre los que podemos señalar a Ahlwalia (1976), Fields (1989) Datt y Ravallion (1992 y 1993), Kakwani (1993) Fishlow (1996), Bourguignon (1996 y 2004), Lal y Myint (1998), Goudi y Ladd (1999) y Ravallion (2001). Generalmente, los trabajos analizan el impacto del crecimiento económico y el nivel de equidad sobre la reducción de pobreza a través de estudios longitudinales con microdatos, en los que tratan de analizar empíricamente la sensibilidad de esta última ante los cambios en renta per cápita y algún índice de inequidad, usualmente el coeficiente de Gini o el índice de Atkinson. Por ejemplo, Kakwani (1993) realiza un análisis para el caso concreto de Costa de Marfil, donde se comprueba por separado la sensibilidad de la pobreza ante cambios en el ingreso promedio y en su distribución, utilizando distintos índices de pobreza para comprobar la elasticidad de cada uno de ellos ante las variaciones mencionadas. Asimismo, Datt y Ravallion realizan un análisis similar, a través de un estudio comparado de Brasil e India durante los años 80, en el que llegan a la conclusión de que "Los efectos del crecimiento y la distribución sobre la pobreza fueron bastante desiguales en el tiempo en ambos países, y los efectos de las casos de crecimiento negativo fueron notablemente diferente entre los dos", Datt y Ravallion (1992, p. 294). 


\section{LA MEDICIÓN DEL BIENESTAR ECONÓMICO A TRAVÉS DE LAS MACROMAGNITUDES DE LA CONTABILIDAD NACIONAL}

Como vemos, este es un debate abierto, con algunos puntos de consenso y disparidad de criterios en otros, en los que los datos juegan un papel primordial, ya que varios de los estudios insisten en que la robustez de estos análisis se ve mermada por la calidad y disponibilidad de los mismos.

\subsection{El bienestar económico en países emergentes y de bajo ingreso}

Hemos comprobado que el análisis de la pobreza difiere de manera importante según la delimitación tomada en consideración, es decir, del concepto de pobreza objeto de estudio. La pobreza absoluta o extrema es una noción muy diferente de la pobreza relativa y, por tanto, no cabe esperar que los resultados sean análogos. La pobreza absoluta implica una situación de indigencia, de privación de las necesidades básicas, de modo que, para establecer las líneas de pobreza absoluta, se suelen emplear indicadores que permitan realizar comparaciones a nivel internacional, como los diseñados por Organismos Internacionales. De hecho, uno de los más populares es la trazada por el Banco Mundial, que fija en un $1,25 \$ 20$ per cápita al día el valor del ingreso mínimo que una persona necesita para no considerar que se encuentra en una situación de pobreza extrema; a esta calificación se añade la de pobreza moderada, dentro de la que se sitúan quienes obtienen unos recursos inferiores a $2 \$$ PPP21. Sin embargo dicho criterio puede resultar un tanto simplista, además de correr el riesgo de caer en la obsolescencia, lo que parece exigir la actualización de las mismas en función de las circunstancias y el contexto actuales. Pero, además, existen otros muchos estudios que tienen por objeto, precisamente, la elaboración de líneas de pobreza absoluta. Una de las primeras investigaciones es la debida a Rowntree (1901), que fijaba el umbral de pobreza en función del valor de una cesta compuesta por una serie de productos básicos, cuya principal crítica se ha dirigido a la subjetividad asociada a la elección de los productos que componen la cesta destinada a satisfacer dichas necesidades. De hecho, aún en la actualidad, y a pesar de que existe un conjunto de bienes y servicios sobre los que no se discute su carácter esencial, no se ha logrado alcanzar un alto nivel de consenso sobre otros muchos y, en consecuencia, la selección final "tiende a depender de la forma de vida de una sociedad concreta y, por tanto, introduce cierta relatividad en la supuesta medida de pobreza absoluta"22. Por su parte, cabe destacar otros trabajos como el de Orshanski 1965 y 1969, en donde se elabora una metodología de medición de la pobreza bajo la premisa de que "el gasto en alimentación de los hogares debe considerarse como una proporción constante del gasto total". La línea de la pobreza estaría formada por una cesta de alimentos básicos valorados a precios de mercado, cuyo coste total se multiplicaría por un factor obtenido como el inverso del peso relativo de los alimentos en el gasto total de una familia media. A pesar de su profusa utilización en Estados Unidos, también se han formulado algunas objeciones a dicha línea, como la derivada de la aplicación de la ley de Engel, por la que se refleja la relación inversa entre crecimiento económico de un país y la proporción del gasto

20.- Posteriormente, estos umbrales se han actualizado a 1,90 \$ y 3,10 \$ PPPs diarios, respectivamente.

21.- Lógicamente, el poder adquisitivo de un dólar es diferente según el país que se tome en consideración. Por eso, las líneas deben ajustarse para tener en cuenta la paridad de poder adquisitivo (PPA) de cada país.

22. - La cesta de alimentos se diseñó por el Departamento de Agricultura de los Estados Unidos, con base en la relación entre los estándares de nutrición y los patrones de consumo de una familia americana. 
en alimentación respecto del total, lo que implica que no siempre una menor proporción de gasto en alimentación equivalga a una mayor proximidad a una situación de pobreza, no cumpliendo así con los requisitos necesarios para ser una medida de pobreza absoluta pura.

Además de las señaladas, se pueden citar líneas de pobreza absoluta cuya utilización es más ocasional, como es el caso del método de las Necesidades Básicas Insatisfechas (NBI) en América Latina; instrumento que se ha empleado para evaluar la pobreza extrema, atendiendo tanto a las características del hogar (vivienda, habitabilidad, acceso a agua potable, luz, etc.) como a los rasgos demográficos y de bienestar del mismo (escolarización de los niños, número de miembros y nivel educativo, asistencia sanitaria, etc.), de modo que los hogares que presenten carencias de acceso en alguno de estos ámbitos básicos son calificados como pobres.

En el análisis que realizamos se adopta, sin embargo, el criterio de pobreza absoluta del Banco Mundial, estableciendo un umbral de $2 \$$ diarios, en términos PPP, teniendo en consideración las diferencias entre los niveles de vida de los distintos países, para lo cual utilizamos la información de la base de datos de Indicadores de Desarrollo Mundial del Banco Mundial, correspondientes al año 201123, ajustando a dólares internacionales, que utilizan tasas de Paridad de Poder Adquisitivo, de manera que cada dólar permite comprar en el país estudiado una cantidad semejante de bienes y servicios, lo que posibilita la comparación del bienestar de los habitantes en términos reales (ICP Banco Mundial) ${ }^{24}$. A pesar de las deficiencias mencionadas de dicho indicador, en la actualidad este es el único con acceso a una base de datos con suficiente información y comparabilidad como para poder obtener conclusiones sólidas, motivo por el cual se ha ponderado la disponibilidad y conveniencia por encima de los aspectos comentados anteriormente. Asimismo, como indicador de la equidad en la distribución se utiliza el coeficiente de Gini, tanto por los aspectos referidos a su preferencia, mencionados en el tercer epígrafe, como de nuevo, por la disponibilidad y posibilidades de establecer comparaciones robustas.

Por tanto, utilizando información de 24 países de bajo o mediano ingreso se realiza un análisis de corte transversal a través de un diagrama de dispersión que considera tres variables, pobreza absoluta en el eje de abscisas, coeficiente de Gini en el eje de ordenadas y renta per cápita, explicada a través del tamaño de las esferas. Como podemos observar, los países cuyo diámetro es inferior registran valores de pobreza absoluta superior, alejándose del origen de abscisas y los mayores se encuentran más próximos al mismo, lo que indica que los países con mayor renta cuentan con una proporción menor de población en pobreza absoluta. En cambio, no podemos establecer el mismo análisis respecto a las ordenadas, de manera que no se establece una relación clara entre el coeficiente de Gini y la renta per cápita. Es decir, mayores niveles de PIB per cápita parecen influir directamente sobre 


\section{LA MEDICIÓN DEL BIENESTAR ECONÓMICO A TRAVÉS DE LAS MACROMAGNITUDES DE LA CONTABILIDAD NACIONAL}

las tasas de pobreza absoluta, lo que evidenciaría una relación inversa entre ambas variables. Como cabría esperar, el crecimiento económico se comporta como una condición necesaria, aunque no suficiente para reducir la pobreza absoluta de los países. Respecto a la equidad en la distribución de los ingresos, se observa que la relación entre el coeficiente de Gini y el PIB per cápita es menos sólida y que, de hecho, no hay un patrón definido. Otro aspecto a reseñar es que los países latinoamericanos presentan valores más altos de inequidad que los países más pobres de Europa, e incluso que los países de África y Asia, aun considerando el caso de aquellos con mayor renta per cápita, como Argentina y especialmente Chile. Este análisis concuerda con aquellos estudios como el de Fields (1995), que señala la mayor desigualdad existente en los países latinoamericanos, con respecto a otras áreas geográficas. Por su parte, Sudáfrica es un valor extremo, en el que un nivel de renta muy superior a la de otros países de los considerados se relaciona con una alta inequidad. Respecto al análisis de pobreza absoluta, los países africanos son los que registran mayores tasas, constituyendo tanto Níger como Senegal valores extremos de la serie, muy alejados de los demás.

\section{Gráfico 1. Relación renta per cápita, pobreza absoluta e inequidad}

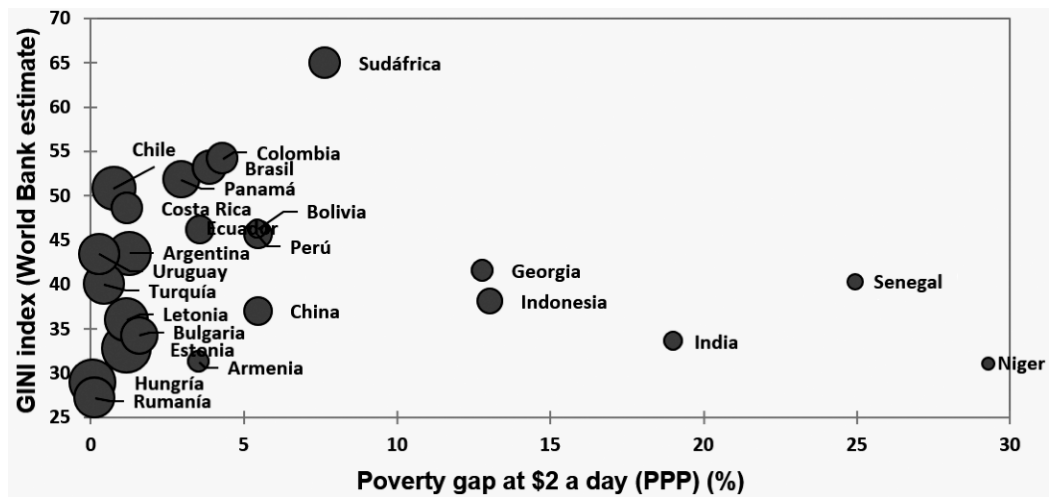

FUENTE: Elaboración propia.

Desglosando los datos, podemos observar, de manera separada, a través de los gráficos de doble barra siguientes, la correspondencia de la renta per cápita, tanto con la pobreza absoluta, como proporción de la población por debajo de la línea de 2 \$ diarios en PPP, como con la inequidad, a través del coeficiente de Gini. Generalmente, aún con excepciones, los niveles más altos de renta se corresponden con tasas inferiores de pobreza, corroborando la relación descrita anteriormente. Sin embargo, se evidencia escasa concordancia con la inequidad, con diferentes situaciones entre países con perfiles similares, señalando la incapacidad explicativa de la renta per cápita en el nivel de equidad de una sociedad. 


\section{Gráfico 2. Relación renta per cápita y pobreza absoluta} (línea de $2 \$$ diarios en PPP)

Poverty Gap 2\$ a day (PPP)

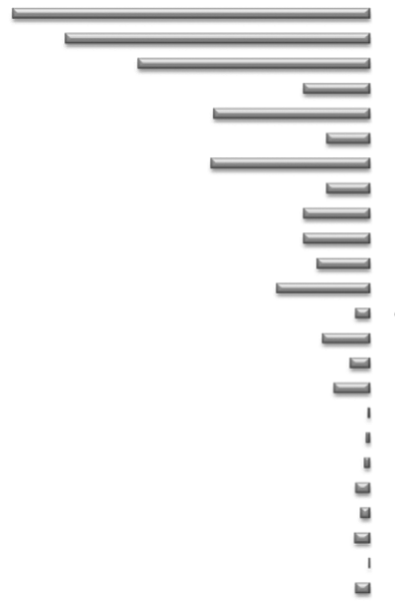

\author{
GDP per cápita
}

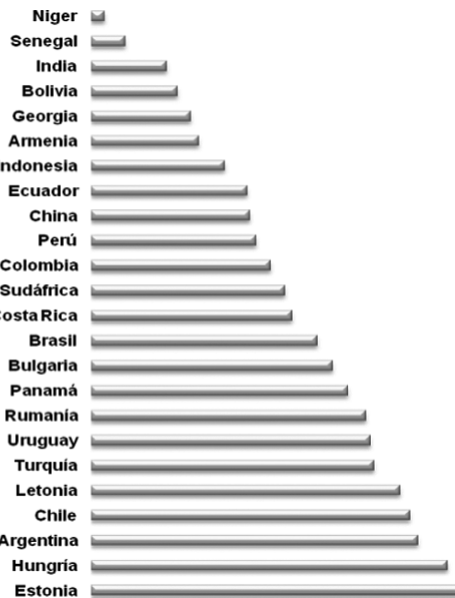

FUENTE: Elaboración propia. Datos Banco Mundial.

Por último, se presenta una regresión de MCO bivariante, que nos ayudará a ilustrar la relación existente entre nivel de renta per cápita y pobreza absoluta. Para ello se han utilizado los mismos datos del Banco Mundial, referenciados al año 2011, con lo que el análisis es puramente transversal. Los resultados detallados se presentan en el ANEXO 1. Una razón de incluir no linealidad en la relación bivariante planteada, tomando logaritmos en la variable exógena, es obtener un modelo sin problemas de eficiencia al acercarse a la varianza constante y normalidad en el error, así como a la independencia de las unidades de medida, teniendo en cuenta que los datos de PIB están expresados en US\$ PPP's y la pobreza absoluta en porcentajes. El coeficiente de la variable exógena entre la centena: $\beta_{1} / 100$, nos muestra, ceteris paribus, que cuando incrementa la pobreza absoluta en una unidad de medida el PIB per cápita aumenta un $1 \%$, por lo que en el modelo especificado, un $1 \%$ más de renta per cápita explicará una reducción de 0,094 unidades pobreza, que al estar expresado en proporción de población pobre por debajo de la línea de pobreza establecida, supondrá una reducción del $0,094 \%$.

$$
P O B R_{A B S i}=92,95-9,370 \log P I B_{P C i}+U_{i} \quad R^{2}=0,854
$$

$\left({ }^{*}\right)$ Resultado significativo al $1 \%$ de nivel de significación. Los números entre paréntesis corresponden a los errores estándar robustos a la heteroscedasticidad. 


\section{LA MEDICIÓN DEL BIENESTAR ECONÓMICO A TRAVÉS DE LAS MACROMAGNITUDES DE LA CONTABILIDAD NACIONAL}

Los resultados indican que mayores niveles de renta se corresponden con menores proporciones de pobreza, ya que el signo del coeficiente es negativo, siendo dicha influencia además significativa $(c<0,001)$. Por otra parte, los contrastes de diagnosis determinan que hay evidencia empírica de inexistencia de mala especificación (RESET) y anormalidad de los residuos (ver ANEXO 1). En cuanto a la corrección del sesgo de los contrastes debido a la heteroscedasticidad, se han utilizado desviaciones típicas robustas.

\section{Gráfico 3. Relación logarítmica GDPPC y pobreza absoluta}

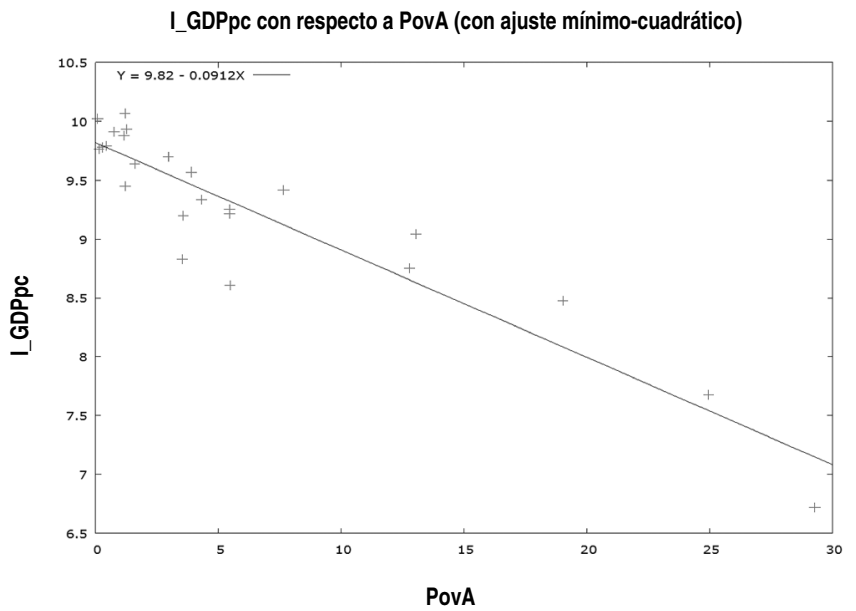

Las conclusiones obtenidas respecto a la variable "pobreza absoluta" se corresponden con los alcanzados por la mayoría de los autores señalados en la revisión bibliográfica comentada. Los estudios de Kakwani (1993), Datt y Ravallion, 1993, Bell y Rich (1994), entre otros, confirman que el crecimiento económico coadyuvaba a la reducción de la pobreza absoluta. Asimismo, Ravallion (2001), en su libro "Growth, inequality, and poverty: looking beyond averages", analizaba, sobre la base de 117 casos entre las encuestas de hogares, elaboradas entre los ochenta y los noventa (2001, p. 1808), y con una cobertura de 47 países en desarrollo, cómo impactaban sobre la pobreza absoluta distintas variaciones de la renta media de los hogares y de la desigualdad, estableciendo una matriz de casos que se retrata a continuación. Ravallion consideraba como índice de pobreza absoluta la línea de $1 \$$ (dólares internacionales PPP 1993) diarios y como indicador de medición de la desigualdad, el coeficiente de Gini. Como resultado, de todos los casos estudiados, el $57 \%$ experimentaron crecimiento económico que se traducía en menores tasas de pobreza absoluta, de manera que el autor concluía que "Incluso en los países en los que la desigualdad está aumentando con el crecimiento del nivel de vida promedio, la pobreza está disminuyendo en promedio. Pero por lo general cae a un ritmo mucho más lento que en los países que experimentan un crecimiento más equitativo" Ravallion (2001, p. 1808). Es decir, los incrementos de la renta media se corresponderían con disminuciones de la pobreza 
absoluta, independientemente de las variaciones que se produjesen en la distribución de la renta, aunque, evidentemente, un incremento de la equidad se traducía para aquellos países que veían reducidos sus ingresos promedios en un aumento inferior de la pobreza y para los que incrementaban la renta, en una mayor reducción de la misma.

Respecto a la relación entre renta per cápita y desigualdad, se puede observar como el nivel de renta per cápita no parece explicar la desigualdad en la distribución de la renta. De nuevo, nos remitimos a la bibliografía comentada y la falta de consenso existente suscitando un intenso debate que, difícilmente, soporta una teoría de disminución sistemática de la inequidad a través del crecimiento económico, sino distintos tipos de relaciones, ya explicadas, que conforman variadas conclusiones sobre este análisis, que en gran parte de los estudios más recientes, tienden a confirmar que el crecimiento por sí solo no puede mejorar la distribución inicial en una sociedad determinada. Estas conclusiones son visibles de manera descriptiva a través de la relación mostrada en los gráficos de barras y de dispersión siguientes. La regresión por mínimos cuadrados tampoco evidencia relación entre ambas variables $(p>|0,10|=0,4368)$ (los resultados detallados se presentan en el ANEXO 1).

\section{Gráfico 4. Relación renta per cápita y desigualdad}

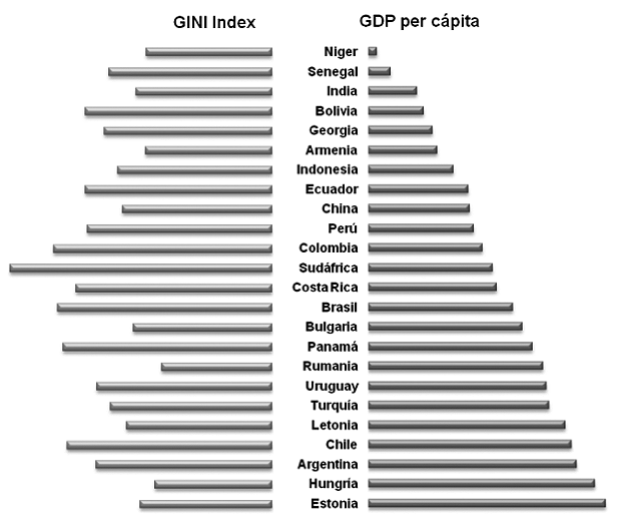

FUENTE: Elaboración propia. Datos Banco Mundial. 


\section{LA MEDICIÓN DEL BIENESTAR ECONÓMICO A TRAVÉS DE LAS MACROMAGNITUDES DE LA CONTABILIDAD NACIONAL}

\subsection{Estudio de la inequidad y la pobreza relativa en economías avanzadas 25}

Como explicábamos, la pobreza relativa es un concepto totalmente distinto, que delimita la situación de la población pobre a un lugar geográfico y un tiempo determinados. Es decir, no es lo mismo un pobre relativo actual que un pobre relativo hace cincuenta años, como tampoco existe analogía entre la pobreza relativa de una economía avanzada con la existente en países de bajo ingreso. Así, la noción de pobreza relativa se relaciona con la noción de inequidad, admitiendo la preexistencia de la misma, derivada de su propia acotación. La construcción de estos umbrales se realiza a través de promedios estadísticos, generalmente la media o la mediana ${ }^{26}$, y tomando en consideración un porcentaje de estos, dividiendo a la unidad poblacional. Lógicamente, es un criterio arbitrario, pues no existe razón alguna que priorice la elección de un porcentaje determinado sobre el resto ni orienta sobre qué variable utilizar. De esta manera, solo en una sociedad situada en la línea de equidad absoluta de la Curva de Lorenz -en la que todos los individuos obtuvieran exactamente la misma rentadesaparecería la pobreza relativa, lo que resulta absolutamente utópico, especialmente en una economía capitalista de mercado, como son la inmensa mayoría de las sociedades actuales. Es más, ni tan siquiera esto supondría variación alguna si la distribución inicial es ya inequitativa, pues un incremento de renta proporcionalmente distribuido no variaría la cantidad de pobreza relativa, aunque sí alteraría la cuantía determinada como umbral anteriormente. Para algunos autores cabría la opción de equiparar esta noción a la de exclusión social, concepto que se juzga diferente y explica una realidad distinta, aunque, en ocasiones, pueda ser coincidente. Como señalan Martínez y Ayala (2001), la exclusión es una "noción dinámica y multidimensional", en la que "las personas pueden ser pobres sin estar excluidas y viceversa" Feres y Villatoro (2012) citan Atkinson (1998) y Atkinson et al. (2005).

Por tanto, las conclusiones obtenidas en el apartado anterior son únicamente consistentes para el concepto delimitado en él, "la pobreza absoluta", requiriéndose un análisis distinto si la pretensión es el estudio del impacto de las variaciones en el ingreso promedio de una sociedad sobre la pobreza relativa de la misma.

Utilizando un panel de países construido a partir de la base de datos de la $O C D E^{27}$, se ha analizado la relación existente entre el nivel de renta per cápita y la proporción de población en pobreza relativa después de transferencias e impuestos, en base a 104 observaciones, conformadas por 13 países desde 2004 a 2011 (datos en US PPP,s). Realizando una regresión de efectos fijos no hallamos evidencia de relación al ser esta estadísticamente no significativa, tanto si realizamos una regresión puramente lineal como si incluimos no linealidad en la relación tomando logaritmos. en cuyo caso los signos si bien son los indicados, no hay significatividad evidente. ${ }^{28}$

25.- Según terminología FMI.

26. - La mediana ha venido a sustituir a la media con el objetivo de evitar las distorsiones de los valores extremos y el error muestral.

27.- OECD.stat

28. - Asimismo, se ha especificado un modelo para comprobar dicha relación tomando como variable de control la distribución de la renta a través del coeficiente de Gini y en este caso obtenemos significatividad en la influencia de la variable exógena "GINl" sobre la pobreza relativa, mostrando una relación positiva entre ambas variables. Sin embargo, la variable exógena "logPIBpc", ahora posee un signo que no es el esperado y además continúa sin ser significativa en la explicación de la pobreza relativa. 
Se evidencia, por tanto, una diferencia entre ambos conceptos de pobreza y en como el PIBpc influye en ambas realidades. Podemos comprobar que el incremento de la renta promedio de una sociedad no supone por sí sola un aumento del bienestar económico de los individuos que la componen y que la manera en que dichos ingresos se distribuyen es la pieza clave para que su incremento altere la situación de partida en beneficio de los más necesitados, lo que se considera como crecimiento "pro-poor", (a favor de los pobres). Las condiciones iniciales, el tipo de ingresos registrados y la acción del Sector Público a través de las políticas públicas parecen delimitar los aspectos más relevantes para diferenciar los distintos resultados del crecimiento económico. Tampoco esta terminología encuentra una definición que goce del consenso académico, ya que, aunque parece superada la noción del efecto derrame (spillover) o efecto goteo (trickle down), por el que, cualquier incremento de la renta, acaba beneficiando a las capas de menor ingreso de la sociedad (amparada en trabajos como el de Dollar y Kraay (2000)), siguen existiendo diversas versiones sobre los efectos del crecimiento y la redistribución, así como el valor que se le atribuye a cada una de estas variables, en la reducción de la pobreza. En este sentido se encuentran posturas distintas, partiendo de consideraciones como la de Ravallion y Chen (2001), que admiten una noción absoluta del mismo, en el que el crecimiento es pro-pobre si consigue reducir la pobreza, lo que, como hemos comprobado sucede generalmente, hasta en conceptos más restringidos y relativos, como el aceptado por Kakwani y Pernia (2000), que supone que únicamente será pro-pobre cuando reduzca la pobreza y la desigualdad, beneficiando relativamente más a los pobres. ${ }^{29}$

\section{5.- La renta disponible ajustada y el papel redistribuidor del sector público}

Centrándonos en este último aspecto, la relevancia dada en los análisis precedentes a la distribución del ingreso presume la preponderancia del papel redistributivo del Estado en la mejora del bienestar económico de una sociedad. De hecho, podemos comprobar, a través de un simple análisis descriptivo de las cifras, que la realidad de una sociedad difiere enormemente cuando las políticas sociales están consolidadas en un país. En los gráficos adjuntos se analizan, en primer lugar, las tasas de pobreza en algunos países de la OCDE seleccionados antes de la acción del estado protector y después de la misma, incorporando en el primer caso la proporción de individuos que en el año 2011 se encontraban por debajo del $60 \%$ de la renta disponible equivalente (línea pobreza relativa OCDE) y en el segundo, el porcentaje resultante, una vez distribuidas las transferencias y gravados los impuestos, en la labor redistributiva los gobiernos, observando que existe una importante diferencia entre

29.- Una explicación amplia de la evolución del término pro-poor puede encontrarse en MEDINA, F. y GALVÁN, M. (2014): “¿Qué es el crecimiento pro-pobre? Fundamentos teóricos y metodologías para su medición", Series Estudios estadísticos, $n^{\circ} 89$, Cepal. 


\section{LA MEDICIÓN DEL BIENESTAR ECONÓMICO A TRAVÉS DE LAS MACROMAGNITUDES DE LA CONTABILIDAD NACIONAL}

ambas tasas. En el segundo gráfico comprobamos los niveles de equidad en los mismos países analizados, a través del coeficiente de Gini, antes y después de transferencias e impuestos. Es fácilmente apreciable la diferencia de ambas variables cuando se tienen en cuenta los ingresos de mercado o los ingresos disponibles. Así lo confirma un estudio de la OCDE realizado en el año 2011, en el que demostraba que la inequidad disminuía en un $25 \%$ de media, cuando se estimaba el coeficiente de Gini sumando las transferencias públicas en efectivo y restando los impuestos sobre la renta y las contribuciones a la Seguridad Social. Asimismo, confirmaba que dicho efecto variaba de unos países a otros, siendo especialmente importante en los Países Nórdicos, Alemania y Bélgica, mientras que Chile, Corea o Estados Unidos sufrían una disminución de la inequidad por debajo de la media 30.

\section{Gráficos 5 y 6. Tasa de pobreza relativa 31 y coeficiente de Gini de los países OCDE a ingresos de mercado e ingresos disponibles}

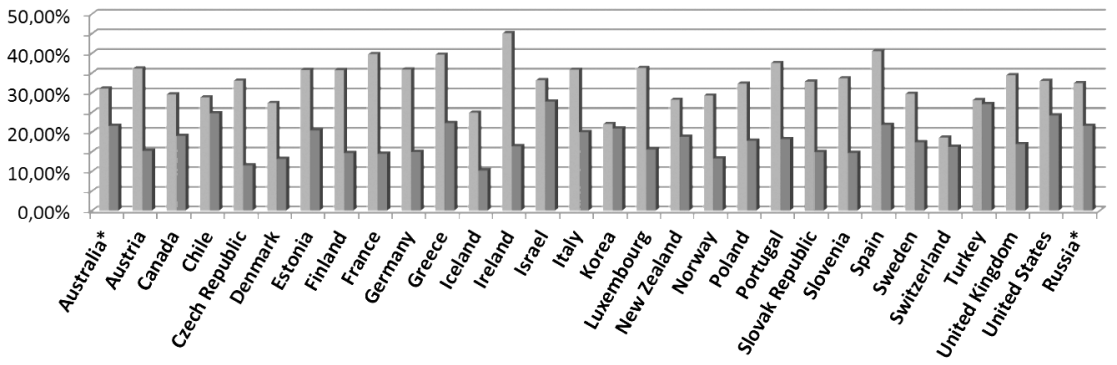

—ANTES DE IMPUESTOS Y TRANSFERENCIAS DESPUÉS DE IMPUESTOS Y TRANSFERENCIAS

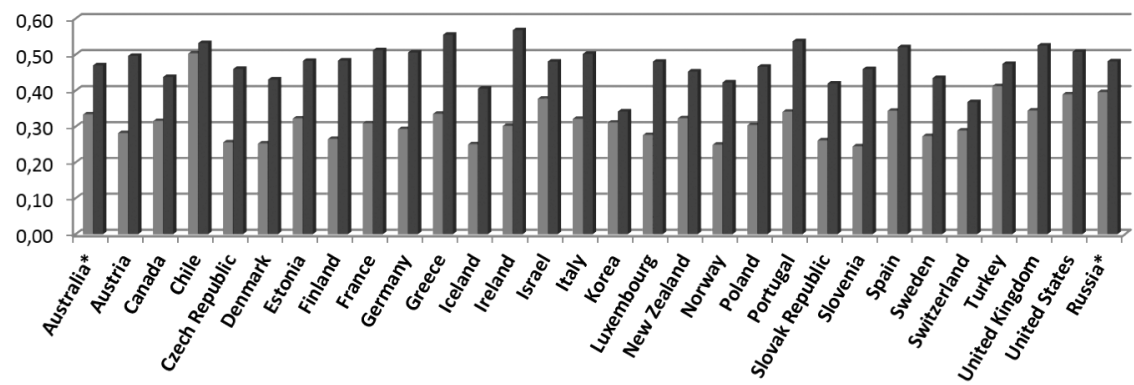

- DESPUÉS DE IMPUESTOS Y TRANSFERENCIAS

- ANTES DE IMPUESTOS Y TRANSFERENCIAS

FUENTE: Elaboración propia y OCDE.

30.- OCDE (2011): "An Overview of Growing Income Inequalities in OECD Countries: Main Findings" (p. 36).

31.- $60 \%$ renta disponible equivalente (línea OCDE). 
Los estudios sobre el impacto del crecimiento económico en la reducción de la pobreza y la inequidad utilizan, generalmente, el PIB per cápita como indicador de la renta promedio de un país, sin embargo, consideramos que los resultados pueden diferir si la medida de ingreso utilizada se aproxima mejor a la noción de bienestar, por lo que procede realizar una revisión de los agregados incluidos en los sistemas de cuentas nacionales, así como en la obtención de dichos índices a través de su contabilización. En este sentido, el manual de los Sistemas de Cuentas Nacionales 2008, reconoce en el apartado $\mathrm{C}$ del capítulo 1 que los agregados resultantes del marco central "han adquirido identidad propia" reconociéndose por "Ios analistas, los políticos, la prensa, la comunidad empresarial y la opinión pública como indicadores sumarios y globales de la actividad económica y del bienestar". Sin embargo, se dice explícitamente que éste no es su objetivo último ni primero, lo que confirma en el apartado $\mathrm{H}$ del mismo capítulo, en el cual reflexiona que "... a menudo se considera el PIB como una medida del bienestar, pero el SCN no adopta esta posición, y de hecho son varias las convenciones del SCN contrarias a una interpretación de las cuentas en términos de bienestar". Asimismo observa que, "(...) aunque algunos aspectos identificadores del bienestar son susceptibles de incluirse en el SCN, otros pueden permanecer fuera del mismo de manera permanente, pues la finalidad de dicho sistema se aparta claramente de la medición del bienestar" (SCN 2008).

No obstante, apartándose de dicha afirmación, las dos últimas versiones de los dos documentos madre parecen haber seguido una tendencia divergente, insertando dentro de sus recomendaciones o exigencias (según la versión referida), herramientas e indicadores cuya finalidad parece aproximarse a la medición del bienestar. En este ámbito, una herramienta de gran interés para dicha medición, al constituir una posibilidad abierta para analizar algunas nociones de utilidad general, relacionadas con el bienestar y el desarrollo, que por su compleja cuantificación, se desarrollan fuera del marco central de los sistemas de cuentas, son las denominadas "Cuentas Satélite". La función principal de las mismas es la de ampliar la información disponible, a través de la elaboración de tablas y cuentas que responden a necesidades específicas y que se desarrollan de manera separada del núcleo de los sistemas de cuentas. Como beneficios derivados de su utilización destacan: la posibilidad de dar mayor detalle en determinadas actividades, añadir información no monetaria sobre aspectos clave y modificar conceptos básicos para obtener resultados paralelos de ciertas rúbricas pero, sin duda, una particularidad en el tema que nos ocupa, es la de dar un tratamiento diferenciado, aunque inserto en las cuentas nacionales, a cuestiones relacionadas con el bienestar o el desarrollo, como es el caso de las cuentas medioambientales, las cuentas de protección social, la producción de los hogares y las cuentas del trabajo y las matrices de contabilidad social. Estas cuentas suponen un paso importante hacia la inclusión de la medición del bienestar en la contabilidad nacional y denotan la tendencia actual a ligar la actividad económica con su repercusión sobre la calidad de vida de los ciudadanos.

Asimismo, desde las revisiones SCN93 y SEC95 se añade a la lista de agregados la Renta Disponible Ajustada de los Hogares, magnitud de gran interés para el propósito de medición del bienestar económico, de tal manera que, para su cálculo, se parte de la Renta Disponible de los hogares, que a su vez se obtiene detrayendo aquellas partidas de las que no pueden disponer los agentes, es decir los impuestos pagados por los mismos, tanto de manera directa como indirecta y tanto por 


\section{LA MEDICIÓN DEL BIENESTAR ECONÓMICO A TRAVÉS DE LAS MACROMAGNITUDES DE LA CONTABILIDAD NACIONAL}

los hogares como por las empresas, incluidas las cotizaciones sociales, así como la parte de renta que no se reincorpora al flujo económico por permanecer dentro de la empresa, es decir, los beneficios no distribuidos o ahorro empresarial y añadiendo las transferencias que el sector público realiza a los hogares y que, por tanto, suponen un incremento de los recursos disponibles. De este modo, la Renta Disponible Ajustada se obtiene incrementando dicha cifra con las transferencias sociales en especie recibidas de las administraciones públicas y las ISFLSH. Se consideran transferencias en especie aquellos servicios proporcionados a los hogares para su disfrute individual de manera gratuita sin contrapartida, lo que equivale a recibir una renta por el valor monetario de los servicios consumidos. Dado que las transacciones se reflejan en las cuentas nacionales en términos monetarios, el procedimiento para reflejar dichas transferencias es el registro de un ingreso a los hogares por unos flujos monetarios equivalentes al valor de esas transferencias. Esto afecta, por tanto, de manera positiva a los hogares que las reciban y, en sentido contrario, al sector público y las instituciones sin ánimo de lucro, AA.PP. e ISFLSH, reduciendo el consumo para ambos sectores en un montante equivalente y opuesto, por lo que para el conjunto de toda la economía el valor de la Renta Disponible Ajustada coincide con el de la Renta Disponible. De este modo, cuando se calcula el ahorro de las administraciones públicas, las ISFLSH y los hogares, el tratamiento ajustado arroja el mismo saldo contable de ahorro para cada sector que el tratamiento estándar. Su utilidad reside precisamente en la posibilidad de aproximar la acción redistributiva del sector público a través del sistema fiscal, las transferencias a los hogares y la provisión de bienes preferentes en especie. La inclusión de bienes en especie como parte del ingreso de los hogares es una cuestión en discusión entre los distintos autores. Atkinson (1991) considera que, dado que el ingreso monetario es necesario para la participación en sociedad, el ingreso en especie no debería incluirse en su medición, por otro lado, Scott afirma que "la diferencia entre privación extrema y alivio relativo en economías menos monetizadas radica usualmente en la disponibilidad o ausencia de alguno de estos servicios, más que en un ingreso personal ligeramente superior o inferior". Feres y Mancero (2001, p. 15) citan a Scott (1981). Asimismo, en el informe de la OCDE del año 2011, mencionado anteriormente, se reconocía que, "en la actualidad, los gobiernos realizaban un alto gasto en la provisión de servicios públicos, principalmente, educación, sanidad y servicios de atención, elevándose al 13\% del PIB de gasto promedio en los países OCDE, lo que suponía, a pesar de que el objetivo principal de dicha provisión no fuese la redistributiva, sino la de solucionar un fallo de mercado en la provisión de dichos bienes preferentes, una mejora importante de la distribución, incrementando la equidad en un $20 \%$ de promedio, medido a través del coeficiente de Gini". Por último, el informe realizado por Stiglitz, Fitoussi y Sen, por encargo de Sarkozy en el año 2009, recomendaba que en la medición de la desigualdad se tuviesen en consideración los bienes preferentes en especie, pues los mismos contribuían indefectiblemente a la mejora del bienestar de aquellos que pudiesen costear por sí mismos dichos servicios.

Dado que la diferencia entre la obtención de bienes y servicios provistos de manera gratuita, ya sea por el sector público o por organizaciones sin ánimo de lucro y la necesidad de pagar dicho consumo requiere de un desembolso monetario, consideramos que es indispensable considerar el valor de dicha oferta a la hora de establecer cuál es el bienestar económico de una sociedad. De hecho, 
uno de los grandes hitos del siglo XXI es la consolidación del Estado del Bienestar, logro social que ha perfilado grandes diferencias entre distintas naciones en función del desarrollo del mismo.

Por tanto, pasamos a comprobar cuál es la influencia del agregado RDA en términos per cápita sobre los niveles de pobreza relativa e inequidad, analizando si existe un mejor ajuste que en los modelos especificados con el PIBpc como variable exógena. Para ello, tomando un panel de países de la OCDE realizamos una regresión de efectos fijos obteniendo que:

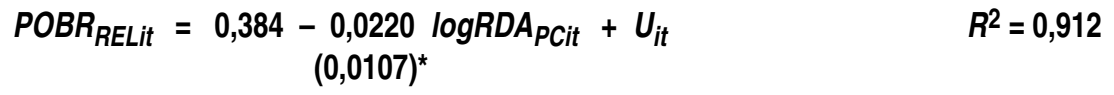

(*) Resultado significativo al 5\% de nivel de significación. Los números entre paréntesis corresponden a los errores estándar robustos a la heteroscedasticidad. Los resultados detallados aparecen en el ANEXO 2.

Como podemos comprobar, se logra una mejora notable en la significancia de los parámetros de todas las variables incluidas y el ajuste del modelo. El signo es el esperado y la influencia mostrada por el RDApc sobre la pobreza relativa es estadísticamente significativa, $=0,0019$., lo que manifiesta que mayores niveles de RDA se corresponden con menores proporciones de pobreza relativa. Asimismo, en base a los mismos datos de panel y a través de una regresión de efectos fijos de panel, se ha analizado la relación con la inequidad a través del coeficiente de Gini, obteniendo que:

$$
\text { GINI }_{i t}=\underset{(0,0168)^{*}}{0,835-0,0540 \log R D A_{P C i t}+U_{i t}} \quad R^{2}=0,940
$$

$\left.{ }^{*}\right)$ Resultado significativo al 1\% de nivel de significación. Los números entre paréntesis corresponden a los errores estándar robustos a la heteroscedasticidad. Los resultados detallados aparecen en el ANEXO 2.

Lo que, de nuevo, nos permite afirmar que el nivel de RDApc influye de manera positiva con menor inequidad en la distribución de la renta y que por tanto coadyuva a la mejora del bienestar económico de la sociedad.

Por tanto, parece que la RDApc se perfila como un mejor indicador del bienestar económico que el PIBpc, por cuanto existe una relación más clara entre mayores niveles de renta y menor proporción de pobreza relativa, así como una distribución más equitativa. Sin embargo, consideramos que todavía sería deseable trabajar sobre dicho agregado para aproximarlo a una medida del bienestar de mayor fidelidad, lo cual cabría realizarse a través de su ajuste en función de la equidad y de la sostenibilidad del mismo, mediante la utilización de coeficientes de mejora que ayuden a aproximar ambas dimensiones, lo cual, lógicamente, excede de la intención de este artículo. 
ANEXO 1. MCO, usando las observaciones $1-24^{*}$

\begin{tabular}{|lccccc|}
\hline POBR $_{\text {ABS }}$ & Coeficiente & Desv. Típica & Estadístico $t$ & Valor $p$ & \\
\hline const & 92.9502 & 7.12591 & 13.0440 & $<0.0001$ & ${ }^{* * *}$ \\
I_GDPpc & -9.37492 & 0.725378 & -12.9242 & $<0.0001$ & ${ }^{* * *}$ \\
\hline GINI & Coeficiente & Desv. Típica & Estadístico $t$ & Valor $p$ & \\
\hline const & 23.5264 & 23.0362 & 1.0213 & 0.3182 \\
I_GDPpc & 1.96559 & 2.48167 & 0.7920 & 0.4368 \\
\hline
\end{tabular}

*Desviaciones típicas robustas ante heterocedasticidad, variante HC1

\section{ANEXO 2. Efectos fijos, utilizando 104 observaciones}

\begin{tabular}{|c|c|c|c|c|c|}
\hline \multicolumn{6}{|c|}{ Relación POBrel/LPIBpc/GINI } \\
\hline$P O B r$ & Coeficiente & Desv. Típica & Estadístico $t$ & Valor $p$ & \\
\hline const & 0.163808 & 0.0226653 & 7.2273 & $<0.0001$ & *** \\
\hline PIBpc & $2.29589 e-07$ & $8.25031 e-07$ & 0.2783 & 0.7814 & \\
\hline POBr & Coeficiente & Desv. Típica & Estadístico $t$ & Valor $p$ & \\
\hline const & 0.32751 & 0.184398 & 1.7761 & 0.0791 & * \\
\hline I_PIBpc & -0.0154648 & 0.0181168 & -0.8536 & 0.3956 & \\
\hline I.POBr & Coeficiente & Desv. Típica & Estadístico $t$ & Valor $p$ & \\
\hline const & $-1,08271$ & 1.05981 & -1.022 & 0.3097 & \\
\hline$I P I B p c$ & $\begin{array}{l}-0.0697195 \\
\end{array}$ & 0.104125 & -0.6696 & 0.5048 & \\
\hline $\mathrm{POB}_{r}$ & Coeficiente & Desv. Típica & Estadístico $t$ & Valor $p$ & \\
\hline const & -0.457946 & 0.175234 & -2.6133 & 0.0105 & ** \\
\hline I_pibpc & 0.0411398 & 0.0158378 & 2.5976 & 0.0110 & ** \\
\hline ginip & 0.691466 & 0.0889942 & 7.7698 & $<0.0001$ & *** \\
\hline
\end{tabular}

Relación RDApc/POBrel / RDA-GINI

\begin{tabular}{|lccccc|}
\hline POBrel & Coeficiente & Desv. Típica & Estadistico $t$ & Valor $p$ & \\
\hline const & 0.835346 & 0.166612 & 5.0137 & $<0.0001$ & ${ }^{* * *}$ \\
I_RDApc & -0.053743 & 0.0167882 & -3.2012 & 0.0019 & ${ }^{* * *}$ \\
\hline GINI & Coeficiente & Desv. Típica & Estadístico $t$ & Valor $p$ & \\
\hline const & 0.835346 & 0.0976337 & 8.5559 & $<0.0001$ & ${ }^{* * *}$ \\
I_RDApc & -0.053743 & 0.00983731 & -5.4632 & $<0.0001$ & ${ }^{* * *}$ \\
\hline
\end{tabular}




\section{6.- Bibliografía}

ACEMOGLU, D. \& ROBINSON, J.A. (2012): Why Nations Fail: The Origins of Power, Prosperity, and Poverty, Deusto S.A. Ediciones, Barcelona.

ADELMAN, I. \& MORRIS, C.T. (1973): Economic growth and social equity in developing countries, Stanford: Stanford University Press.

AGHION, P. \& HOWITT, P. (1998): Endogenous Growth Theory, Cambridge: MITT Press.

AHLUWALIA, M.S. (1976): "Income distribution and development: some stylized facts", American Economic Review, 66, 128-135.

AHLUWALIA, M., CARTER, N., CHENERY, H. (1979): Growth and poverty in developing countries (revised). Staff working paper SWP 309, Washington, D.C: The World Bank.

ALESINA, A. \& PEROTTI, R. (1994): "The political economy of growth: a critical survey of the recent literature", World Bank Economic Review, 8 (3), 351-371.

ALESINA, A. \& RODRIK, D. (1994): "Distributive politics and economic growth", Quarterly Journal of Economics, 109 (2), 465-490.

ALTIMIR, O. \& PINERA, S. (1979): "Análisis de Descomposición: Una generalización del método de Theil”, Cuadernos de Economía, 16 (48) 207-236.

ANAND, S. \& KANBUR, R. (1993 a): "The Kuznets process and inequality-development relationship", Journal of Development Economics, 40, 25-52.

ANAND, S. \& KANBUR, R. (1993 b): "Inequality and development: a critique", Journal of Development Economics, 41, 19-43.

ATKINSON, A. (1991): "Comparing Poverty Rates Internationally: Lessons from Recent Studies in Developed Countries", World Bank Economic Review, 5 (1), 3-21.

ATKINSON, A. (1998): Social exclusion, poverty and unemployment, CASSE paper, $n^{\circ} 4$.

ATKINSON, A., CANTILLON, B., MARLIER, E. \& NOLAN, B. (2005): Social Indicators, The EU and Social Inclusion, New York: Oxford University Press.

BAREA, J. \& MONZÓN, J.L. (2006): Manual para la elaboración de las cuentas satélite de las empresas de la economía social: cooperativas y mutuas, Comisión Europea, CIRIEC-Internacional.

BARNAJEE, A. \& DUFLO, E. (2003): "Inequality And Growth: What Can The Data Say?", Journal of Economic Growth, 8, 267-299. 
BARRO, R. (1999): Inequality, growth and investment, NBER Working Paper $n^{\circ} 7038$. Cambridge: NBER.

BELL \& RICH (1994): "Rural poverty and agricultural performance in pot-independence India", Oxford Bulletin of Economics and Statistics, 56 (2), 111-133.

BENABOU, R. (1994): Education, Income Distribution, and Growth: the Local Connection, NBER Working Paper 4798. Cambridge: NBER.

BIRDSALL, N., ROSS, D. \& SABOR, R. (1995): "Inequality and growth reconsidered: Lesson from East Asia", World Bank Economic Review, 9 (3), 477-508.

BOOTH, C. (1901): Life and labour of the people in London, Macmillan, Londres. (Charles Booth online archive).

BOURGUIGNON, F. (1994): "Growth, distribution and human resources". In G. Ranis (editor), Route to modern growth: Essays in Honor of Carlos Diaz Alejandro, Baltimore: John Hopkins University.

BOURGUIGNON, F. (1996): "Comment on «Inequality, poverty and growth: Where do we stand»?" Annual World Bank Conference on Development Economics, Washington D.C: World Bank.

BOURGUIGNON, F. (2001): The distributional effects of growth: micro vs. macro approaches, Washington D.C: World Bank y Delta-Paris: Mimeo.

BOURGUIGNON, F. (2002): The growth elasticity of poverty reduction: explaining heterogeneity across countries and time periods, Working Paper 03. Paris: DELTA, Centre National de la Recherche Scientifique.

BOURGUIGNON, F. (2004): "The poverty-growth-inequality triangle", Indian Council for Research on International Economic Relations, Nueva Delhi, 4 de febrero.

CLARK, C. (1937): National Income and Outlay, Ed. Macmillan and Company, London.

CLARKE, G. (1995): "More evidence on income distribution and growth", Journal of Development Economics, 47.

COMISIÓN DE LAS COMUNIDADES EUROPEAS (2009): Comunicación de la Comisión al Consejo y al Parlamento Europeo: Más allá del PIB: Evaluación del progreso en un mundo cambiante, Bruselas.

DATT, G. \& RAVALLION, M. (1993): "Regional disparities, targeting and poverty in India". In M. Lipton \& J. Van Der Graag (eds.), Including the poor, Washington D.C: World Bank.

DAVIES, J.B., SANDSTROM, S., SHORROCKS, A. \& WOLFF, E.N. (2008): The World Distribution of Household Wealth, WIDER Discussion paper $n^{\circ} 3$. Helsinki: UNU-WIDER.

DAWSON, P.J. (1997): "On testing Kuznets 'economic growth hypothesis", Applied Economic Letters, 4, pp. 409-410. 
DEININGER, K. \& SQUIRE, L. (1998): "New ways of looking at old issues: inequality and growth", Journal of Development Economics, 57 (2), 259-287.

DIENER, E. (2000): "Subjective well-being: The science of happiness and a proposal for a national index", American Psychologist, 55, 34-43.

DOLLAR, D. \& KRAAY, A. (2000): Growth is Good for the Poor, World Bank Working Paper, Washington D.C: World Bank.

DOMÍNGUEZ, J. \& CARABALLO, A. M. (2006): "Medición de la pobreza: una revisión de los principales indicadores", Revista de Métodos Cuantitativos para la economía y la empresa, 2, 27-66.

EASTERLY, W. (2003): "A tale of two kuznets curves: inequiality in the Old and New Globalizations", NBER-Pre-Conference on Globalization and Inequality, Cambridge.

EASTERLIN, R.A. (1995): "Will Raising the Incomes of All Increase the Happiness of All?", Journal of Economic Behavior and Organization, 27 (1), 35-47.

EUROSTAT (2013): European System of Accounts 2010, European Comission, UE.

FERES, J.C. \& MANCERO, X. (2001): Enfoques para la medición de la pobreza: breve revisión de la literatura, Estudios estadísticos y descriptivos, Santiago: CEPAL.

FERES, J.C. \& VILLATORO, P. (2012): La viabilidad de erradicar la pobreza: Un examen conceptual y metodológico, Estudios estadísticos y prospectivos, Series $n^{\circ} 78$, Santiago: CEPAL.

FERULLO, H. (2006): "El concepto de pobreza en Amartya Sen", Cultura económica, 66, 10-16.

FIELDS, G.S. (1980): Poverty, inequality and development, Cambridge: Cambridge University Press.

FIELDS, G.S. (1989): "Changes in poverty and inequality in developing countries", World Bank Research Observer, 4, 167-86.

FIELDS, G.S. (1995): "La curva de Kuznets: una buena idea pero...". Cuadernos Económicos de ICE, 61, 59-77.

FIELDS \& JAKUBSON (1994): New evidence on the Kuznets curve, Mimeo, Cornnell University, Ithaca.

FISHLOW, A. (1996): "Inequality, poverty, and growth: Where do we stand?", Annual World Bank Conference on Development Economics, Washington D.C: World Bank.

FOSTER, J. \& SHORROCKS, A. (1991): "Subgroup consistent poverty indices", Econometrica, 59, 687-709.

FOSTER, J. \& SZEKELY, M. (2001): Is Economic Growth Good for the Poor? Tracking Low Incomes Using General Means, Working Paper n ${ }^{\circ} 453$, Washington D.C: Inter-American Development Bank.

FOSTER, J.E., GREER, J. \& THORBERCKE, E. (1984): "A class of decomposable poverty measures", Econometrica, 52 (3), 761-767. 
GOUDIE, A. \& LADD, P. (1999): "Economic growth and poverty inequality", Journal of International Development, 11 (2), 177-195.

HAAGENARS (1991): The perception of poverty, Elsevier Science publishers B.V. Amsterdam.

INSTITUTO NACIONAL DE ESTADÍSTICA (2006): La pobreza y su medición. Presentación de diversos métodos de obtención de medidas de pobreza, Madrid: INE.

JAIN, S. (1975): Size distribution of income: A compilation of data, World Bank.

JAIN, L.R. \& TENDULKAR, S.D. (1990): "Role of Growth and Distribution in the Observed Change in Poverty", Indian Economic Review, 25 (2), 165-204.

JENKINS, S. \& LAMBERT, P. (1997): "Three 'i's of poverty curves, with an analysis of UK poverty trends", Oxford Economics Papers, 49 (3), 317-327.

KAKWANI, N. (1986): Analyzing redistribution policies: a study using Australian data, Cambridge: Cambridge University Press.

KAKWANI, N. (1993): "Poverty and economic growth with application to Côte d'Ivoire", Review of Income and Wealth, Series 39 (2), 121-139.

KAKWANI, N. \& PERNIA, E. (2000): "What is Pro-Poor Growth?", Asian Development Review, 18 (1), 1-16.

KAKWANI, N. (1981): "Welfare Measures: An International Comparison", Journal of Development Economics, 8, 21-45.

KALDOR, N. (1956): "Alternative theories of distribution", Review of Economic Studies, 23 (2), 83-100.

KAPTEYN, A. \& VAN PRAAG, B.M.S. (1976): "A new approach to the construction of family equivalence scales", European Economic Review, 7, 313-335.

KEYNES, J.M. (1936): Teoría general de la ocupación, el interés y el dinero, Fondo de cultura económica de España, Ed. 2006 Madrid.

KING, G. (1696): "Natural and political observations and conclusions upon the state and condition of England". Reeditado en 1973 en P. Laslett (ed.), The Earliest Classics, Farnborough: Gregg International.

KNIGHT, J.B. (1976): "Explaining Income Distribution in Less Developed Countries: A Framework and an Agenda", Oxford Bulletin of Economic and Statistics, 38 (3), 161-177.

KUZNETS, S. (1955): "Economic Growth and Income Inequality", American Economic Review, 45 (1), $1-28$.

KUZNETS, S. (1966): Modern Economic Growth, New Haven, CT: Yale University Press.

LAL, D. \& MYINT, U.H. (1998): The political economy of poverty, equity, and growth: a comparative study, Oxford: Clarendon Press. 
LEONTIEF, W. (1951): The Structure of the American Economy, 1919-1939: An Empirical Application of Equilibrium Analysis, Second Edition, Oxford University Press, New York.

LEÓN, P. \& MARCONI, R.S. (1999): La contabilidad nacional: teoría y métodos, Quito (Abya Yala).

LEQUILLER, F. \& BLADES, F. (2009): Comprendiendo las cuentas nacionales, OCDE, París.

LEWIS, A. (1954): Economic development with unlimited supplies of labor, Manchester, School of Economics and Social Studies.

LIPSEY, R.G. (1981): Introducción a la economía positiva, 12ª Edición 1996, Vicens-Vives. Barcelona.

LUCAS, R.E. (1988): "On the Mechanics of Economic Development", Journal of Monetary Economics, 22, 3-42.

MALINVAUD, E. (1973): Initiation à la comptabilité nationale, Paris : Insee.

MARTÍNEZ LÓPEZ, R. \& AYALA CAÑÓN, L. (2001): "Pobreza y exclusión social en la Unión Europea: La nueva estratificación social en perspectiva", Documentación Social, 123, 245-267.

MEDINA, F. \& GALVÁN, M. (2008): Descomposición del coeficiente de Gini por fuentes de ingreso: Evidencia empírica para América Latina 1999-2005, Estudios estadísticos y prospectivos, Serie Cepal, 63, Santiago: CEPAL.

MEDINA, F. \& GALVÁN, M. (2014): ¿Qué es el crecimiento pro-pobre? Fundamentos teóricos y metodologías para su medición, Estudios estadísticos y prospectivos, Series Cepal, 89, Santiago: Cepal.

MILANOVIC, B. (2005): Worlds Apart, Measuring International and Global Inequality, Princeton y Oxford: Princeton University Press.

Mill, J.S. (1991): On liberty and other essays, Oxford University Press, USA.

NACIONES UNIDAS (2009): System of National Accounts 2008 (2008 SNA), United Nations Statistical Commission (UNSC), New York.

NURKSE, R. (1953): Problems of Capital Formation in Underdeveloped Countries, Oxford University Press, 8th impression 1961, New York.

OCDE (2009): The Global Project on Measuring the Progress of Societies: A Toolkit for Practicioners, Commission on the Measurement of Economic Performance and Social Progress, Paris.

OCDE (2011): An Overview of Growing Income Inequalities in OECD Countries: Main Findings, Divided We Stand. Why Inequality Keeps Rising?, OECD Publishing, Paris.

ORSHANSKY, M. (1965): "Counting the poor. Another look at the poverty profile", Social security bulletin, 28 (1), 3-29, Washington, U.S Department of Health, Education and Welfare.

OSBERG, L. \& SHARPE, A. (2003): "Human Well Being and Economic Well Being: What Values are Implicit in Current Indices?", CSLS Research Reports, 4. 


\section{LA MEDICIÓN DEL BIENESTAR ECONÓMICO A TRAVÉS DE LAS MACROMAGNITUDES DE LA CONTABILIDAD NACIONAL}

OSBERG, L. \& SHARPE, A. (2002): "An index of economic well-being for selected countries", Review of Income and Wealth, 48, 291-316.

PAUKERT, F. (1973): "Income distribution at different levels of development: A survey of evidence", International Labour Review, 108, 97-125.

PETTY, W. (1665): Verbum Sapienti or an Account of the Wealth and Expences of England, and the Method of raising Taxes in the most Equal Manner, Routledge and Thoemmes Antiquarian Books.

PIGOU, A.C. (1912): Wealth and Welfare, Ed. Macmillan and Company, London.

PIGOU, A.C. (1920): La Economía del Bienestar, Traducción al Castellano de Economics of Welfare, Editorial Aguilar, Madrid, 1946.

PONCE ZUBILLAGA, M.G. (2013): "Pobreza y bienestar: una mirada desde el desarrollo", Cuadernos del Cendes, 30 (83), (mayo-agosto), 1-21.

RAM, R. (1988): "Economic development and income inequality: Further evidence on the U-curve hypothesis", World Development, 16 (11), 1371-1386.

RAVALLION, M. (1997): "Good and bad growth: The Human Development Reports", World Development, 25, 631-638.

RAVALLION, M. \& HUPPI, M. (1991): "Measuring Changes in Poverty: A Methodological Case Study of Indonesia during an Adjustment Period", World Bank Economic Review, 5 (1), 57-82.

RAVALLION, M. (1995): "Growth and poverty: evidence for the developing world", Economic Letters, $48,411-417$.

RAVALLION, M. (2001): "Growth, inequality and poverty: Looking beyond averages", World Development, 29 (11), 1803-1815.

RAVALLION, M. \& CHEN, S. (1997): "What can new survey data tell us about recent changes in distribution and poverty?", World Bank Economic Review, 11 (2), 357-382.

RAVALLION, M. \& CHEN, S. (2001): "Measuring pro-poor growth", Economics Letters, 78, 93-99.

RAVALLION, M. \& DATT, G. (1999): When is Growth Pro-Poor? Evidence from the Diverse Experiences of India's States, Policy Research Working Paper, 2263, Washington, D.C: World Bank.

RAVALLION, M. \& HUPPI, M. (1991): "Measuring Changes in Poverty: A Methodological Case Study of Indonesia during an Adjustment Period", World Bank Economic Review, 5 (1), pp. 57-82.

ROBINSON, S. (1976): "A Note on the U Hypothesis relating Income Inequality and Economic Development", American Economic Review, 66 (3), 437-440.

ROMER, P. (1986): "Increasing Returns and Long-Run Growth", The Journal of Political Economy, 94 (5), 1002-1037. 
ROS, J. (2004): La teoría del desarrollo y la economía del crecimiento, México D.F: CIDE y Fondo de Cultura Económica.

ROWNTREE, B.S. (1901): Poverty: A Study of Townlife, London: Palgrave Macmillan.

ROSENTEIN-RODAN, P.N. (1943): "Problems of Industrialisation of Eastern and South-Eastern Europe", The Economic Journal, 53, № 210/211, 202-211.

ROSTOW, W.W. (1952): The process of Economic Growth, W.W. Norton \& Company, Inc., Revised edition 1962.

SAITH, A. (1983): "Development and distribution: a critique of the cross-country U-hypothesis", Journal of Development Economics, 13 (3), 367-382.

SEN, A. (2000): "Las distintas caras de la pobreza", Periódico El País, Versión impresa, 30 de agosto.

SEN, A. (1987): "El nivel de vida", Editorial Complutense, Madrid, Nuevo examen de la desigualdad, Alianza Editorial, 2004.

SEN, A. (2000): Desarrollo y libertad, Oxford: Oxford University Press, Traducción Ed. Planeta, Barcelona, Original: Development as Freedom, Anchor Book, Nueva York.

SEERS, D. (1969): "The Meaning of Development", International Development Review, Institute of Development Studies, 11 (4).

SOLOW, R. (1956): "A contribution to the theory of economic growth", Quarterly Journal of Economics, 70 (1), 65-94.

STIGLITZ, J.E., SEN, A. \& FITOUSSI, J. (2009): Report by the Commission on the Measurement of Economic Performance and Social Progress, Paris: Commission on the Measurement of Economic Performance and Social Progress.

STONE, R. \& MEADE, J. (1942): "The Precision of National Income Estimates", The Review of Economic Studies. 9 (2), 111-125.

TOWNSEND, P. (1979): Poverty in the United Kingdom: A survey of household resources and standard of living, Middlesex: Penguin Books.

TSAKLOGOU, P. (1988): "Development and inequality revisited", Applied Economics, 20, 509-531.

NACIONES UNIDAS (2011): Informe de Desarrollo Humano, Anexo Estadístico, Paris: UNDP.

VAN PRAAG, B.M.S., HAGENAARS, A.J.M. \& VAN WEEREN, J. (1982): "Poverty in Europe", The Review of Income and Wealth, 28 (3), 345-359.

\section{BASES DE DATOS}

BANCO MUNDIAL: http://databank.bancomundial.org/data/home.aspx

OCDE: http://stats.oecd.org/ 